\title{
PATH DECOMPOSITIONS FOR REAL LEVY PROCESSES
}

\author{
Thomas DUQUESNE \\ Université Paris-Sud, 91405 Orsay cedex, France \\ Received 7 June 2001, revised 22 January 2002
}

ABSTRACT. - Let $X$ be a real Lévy process and let $X^{\uparrow}$ be the process conditioned to stay positive. We assume that 0 is regular for $(-\infty, 0)$ and $(0,+\infty)$ with respect to $X$. Using elementary excursion theory arguments, we provide a simple probabilistic description of the reversed paths of $X$ and $X^{\uparrow}$ at their first hitting time of $(x,+\infty)$ and last passage time of $(-\infty, x]$, on a fixed time interval $[0, t]$, for a positive level $x$. From these reversion formulas, we derive an extension to general Lévy processes of Williams' decomposition theorems, Bismut's decomposition of the excursion above the infimum and also several relations involving the reversed excursion under the maximum.

ㄷ 2003 Éditions scientifiques et médicales Elsevier SAS

RÉSUMÉ. - Soit $X$ un processus de Lévy et $X^{\uparrow}$ le même processus conditionné à rester positif. On suppose que 0 est régulier pour $(-\infty, 0)$ et $(0,+\infty)$ par rapport à $X$. Par des arguments simples de théorie des excursions, nous décomposons la loi des trajectoires de $X$ et $X^{\uparrow}$ retournées aux temps d'entrée de $(x,+\infty)$ et de sortie de $(-\infty, x]$. De ces formules de reversion, on déduit une extension au cas des processus de Lévy généraux, des théorèmes de décomposition de Williams, du théorème de décomposition de Bismut de l'excursion au dessus du minimum, ainsi que plusieurs relations faisant intervenir l'excursion sous le maximum retournée.

(c) 2003 Éditions scientifiques et médicales Elsevier SAS

\section{Introduction}

Let $\left(X_{t}\right)_{t \geqslant 0}$ be a real Lévy process, that is a real valued process with homogeneous and independent increments. The supremum (respectively infimum) of $X$ on the time interval $[0, t]$ is denoted by $S_{t}$ (respectively $\left.I_{t}\right)$. We assume that 0 is regular for $(0,+\infty)$ and $(-\infty, 0)$ with respect to $X$. A classical result says that $X-I$ (respectively $X-S$ ) is a strong Markov process for which 0 is regular (see Bingham [5] or Bertoin [4] Chapter 6 for a proof). Let us denote by $L$ (respectively $L^{*}$ ) the local time at 0 of $X-I$ (respectively $X-S$ ): they are uniquely defined up to a multiplicative constant and their normalization is specified in Proposition 2.3.

As Rogers noticed in [17], as soon as the Lévy measure charges the positive numbers, $X-S$ may hit zero by $X$ jumping across the level of its previous maximum. The classical

E-mail address: thomas.duquesne@math.u-psud.fr (T. Duquesne). 
Itô excursion measure of $S-X$ loses the information about this jump. Let us introduce the relevant definition of the excursion measure under the maximum (respectively above the infimum) denoted by $N^{*}$ (respectively $N$ ). It has the property to record the final jump of the excursion, which represents the amount the excursion overshoots when $X$ attains a new maximum (respectively infimum). Let $\left(g_{i}, d_{i}\right), i \in \mathcal{I}$ (respectively $\left.\left(g_{j}, d_{j}\right), j \in \mathcal{I}^{*}\right)$ the excursion intervals of $X-I$ (respectively $S-X$ ) above 0 . We define the excursions above the infimum and under the supremum by

$$
\begin{cases}\omega^{i}(s)=X_{\left(g_{i}+s\right) \wedge d_{i}}-X_{g_{i}}, & i \in \mathcal{I}, \\ \omega^{j}(s)=X_{\left(g_{j}+s\right) \wedge d_{j}}-X_{g_{j}}, & j \in \mathcal{I}^{*} .\end{cases}
$$

Then, the point measures

$$
\sum_{i \in \mathcal{I}} \delta_{\left(L_{g_{i}}, \omega^{i}\right)} \quad \text { and } \quad \sum_{j \in \mathcal{I}^{*}} \delta_{\left(L_{g_{j}}^{*}, \omega^{j}\right)}
$$

are distributed respectively as $\mathbf{1}_{\{l \leqslant \eta\}} \mathcal{N}(d l d \omega)$ and $\mathbf{1}_{\left\{l \leqslant \eta^{*}\right\}} \mathcal{N}^{*}(d l d \omega)$, where $\mathcal{N}$ and $\mathcal{N}^{*}$ are Poisson measures with respective intensities $d l N(d \omega)$ and $d l N^{*}(d \omega)$, and where

$$
\left\{\begin{array}{l}
\eta=\inf \{t \geqslant 0: \mathcal{N}([0, t] \times\{\zeta(\omega)=\infty\}) \geqslant 1\}, \\
\eta^{*}=\inf \left\{t \geqslant 0: \mathcal{N}^{*}([0, t] \times\{\zeta(\omega)=\infty\}) \geqslant 1\right\}
\end{array}\right.
$$

( $\zeta(\omega)$ being the lifetime of the path $\omega$ ). The random variables $\eta$ and $\eta^{*}$ have the same law as respectively $L_{\infty}$ and $L_{\infty}^{*}$, that are exponentially distributed or infinite a.s.

In Section 3, Theorem 3.3 provides a decomposition of the law of the excursion under the supremum reversed at its final jump: More precisely, we decompose the law of $\left(\omega_{\zeta(\omega)}-\omega_{(\zeta(\omega)-s)-} ; 0 \leqslant s \leqslant \zeta(\omega)\right)$ under $N^{*}\left(\cdot \bigcap\left\{\omega_{\zeta}>0\right\}\right)$, in terms of the law of $X$ and its Lévy measure. Theorems 3.2 and 3.1 give similar results for $\left(X_{\tau_{x}}-X_{\left(\tau_{x}-s\right)-} ; 0 \leqslant s \leqslant\right.$ $\left.\tau_{x}\right)$ under $\mathbf{P}\left(\cdot \mid X_{\tau_{x}}>x\right)$ and $\left(X_{\sigma_{x}(t)}-X_{\left(\sigma_{x}(t)-s\right)-} ; 0 \leqslant s \leqslant \sigma_{x}(t)\right)$ under $\mathbf{P}\left(\cdot \mid X_{\sigma_{x}(t)}>x\right)$, where we have set for any $x, t>0$ :

$$
\tau_{x}=\inf \left\{s \geqslant 0: X_{s}>x\right\} \quad \text { and } \quad \sigma_{x}(t)=\sup \left\{s \in[0, t]: X_{s} \leqslant x\right\} .
$$

Williams in [19], and many authors after him, explored the connections between the Brownian motion, the three-dimensional Bessel process and the Brownian excursion (see for instance Pitman [16] and Bismut [6]). Many of these identities in the Brownian case hold in a more general setting for totally asymetric Lévy processes: see Bertoin [2] for a generalized Pitman theorem for spectrally negative Lévy processes and Chaumont [79] for Williams' theorems and Bismut's decomposition in the spectrally positive case. Let us mention that Chaumont has also explored the stable case in detail in [10] and [7], providing several path-constructions and identities concerning the stable meander, the normalised excursion and the stable bridge. In these results the role of the three-dimensional Bessel process is played by the Lévy process conditioned to stay positive. This process, denoted by $X^{\uparrow}$, has been introduced by Bertoin in a general setting (see [3]). Bertoin's construction of $X^{\uparrow}$ is recalled in Section 2.2. We use it in combination with Theorems 3.2 and 3.1 to get in Section 4.1 the generalized 
first Williams' decomposition theorem, then Bismut's decomposition of the excursion above the infimum in Section 4.2 and the second Williams' decomposition theorem in Section 4.3.

Let us explain more precisely these results: For any $t>0$, we define $U_{t}^{*}=X_{L_{t}^{*-1}}$ if $L_{\infty}^{*}>t$ and $U_{t}^{*}=+\infty$ if not. The process $\left(U_{t}^{*} ; t \geqslant 0\right)$ is a subordinator (see Bertoin [4], Chapter 6) and its drift coefficient is denoted by $d^{*}$. A classical result due to Kesten (see [11]) ensures that $\mathbf{P}\left(X_{\tau_{x}}=x\right)>0$ iff $d^{*}>0$. We assume that $d^{*}>0$ and that $X$ does not drift to $-\infty$. Then, we can show that $\sigma_{x}^{\uparrow}=\sup \left\{s \geqslant 0: X_{s}^{\uparrow} \leqslant x\right\}$ is finite a.s. Theorem 4.2 show that

$$
\mathbf{P}\left(X_{\sigma_{x}^{\uparrow}}^{\uparrow}=x\right)=\mathbf{P}\left(X_{\tau_{x}}=x\right)
$$

and that $\left(x-X_{\left(\tau_{x}-s\right)-} ; 0 \leqslant s \leqslant \tau_{x}\right)$ under $\mathbf{P}\left(\cdot \mid X_{\tau_{x}}=x\right)$ has the same law as $\left(X_{s}^{\uparrow} ; 0 \leqslant\right.$ $\left.s \leqslant \sigma_{x}^{\uparrow}\right)$ under $\mathbf{P}\left(\cdot \mid X_{\sigma_{x}^{\uparrow}}^{\uparrow}=x\right)$.

We also prove in Theorem 4.5 a path decomposition of the excursion above the infimum similar to Bismut's decomposition of the Brownian excursion: we show that for any nonnegative measurable functionals $G$ and $D$ on the space of càdlàg paths with a finite lifetime and for any nonnegative measurable function $f$,

$$
\begin{aligned}
& N\left(\int_{0}^{\zeta(\omega)} d t G\left(\omega_{s} ; 0 \leqslant s \leqslant t\right) f\left(\omega_{t}\right) D\left(\omega_{t+s} ; 0 \leqslant s \leqslant \zeta(\omega)-t\right)\right) \\
& \quad=\int_{0}^{+\infty} d x f(x) u^{*}(x) \mathbf{E}\left[G\left(X_{s}^{\uparrow} ; 0 \leqslant s \leqslant \sigma_{x}^{\uparrow}\right) \mid X_{\sigma_{x}^{\uparrow}}^{\uparrow}=x\right] \mathbf{E}\left[D\left(X_{s} ; 0 \leqslant s \leqslant \tau_{-x}\right)\right],
\end{aligned}
$$

where $\tau_{-x}=\inf \left\{s \geqslant 0: X_{s}<-x\right\}$ and where $u^{*}$ is the co-excessive version of the density of the potential measure associated with the subordinator $U^{*}$.

Section 4.3 is devoted to the proof of Theorem 4.10 that can be seen as an analogue for general Lévy processes of the second Williams' decomposition theorem that originally concerns the Brownian excursion split at its maximum. Let us describe our result: For any $x>0$, we set $\tau_{x}^{\uparrow}=\inf \left\{s \geqslant 0: X_{s}^{\uparrow}>x\right\}$. Proposition 4.7 shows that

$$
\mathbf{P}\left(X_{\tau_{x}^{\uparrow}}^{\uparrow}=x\right)>0 \quad \text { iff } \quad d^{*}>0
$$

Let us denote by $X^{\downarrow}$ the process $X$ conditioned to stay negative (that is defined in Section 2.2); we write $\bar{g}(\omega)$ the instant when the excursion $\omega$ attains its maximum. Theorem 4.10 shows the law of $\omega_{\bar{g}(\omega)}$ under $N$ admits a density with respect to Lebesgue measure that we specify. Under $N\left(\cdot \mid \omega_{\bar{g}(\omega)}=x\right)$, the processes $\left(\omega_{s} ; 0 \leqslant s \leqslant \bar{g}(\omega)\right)$ and $\left(\omega_{s+\bar{g}(\omega)} ; 0 \leqslant s \leqslant \zeta(\omega)-\bar{g}(\omega)\right)$ are mutually independent. Furthermore, $x)$;

- the process $\left(\omega_{s} ; 0 \leqslant s \leqslant \bar{g}(\omega)\right)$ is distributed as $\left(X_{s}^{\uparrow} ; 0 \leqslant s \leqslant \tau_{x}^{\uparrow}\right)$ under $\mathbf{P}\left(\cdot \mid X_{\tau_{x}^{\uparrow}}^{\uparrow}=\right.$

- the law of $\left(\omega_{s+\bar{g}(\omega)} ; 0 \leqslant s \leqslant \zeta(\omega)-\bar{g}(\omega)\right)$ is absolutely continuous with respect to the law of $\left(X_{s}^{\downarrow} ; 0 \leqslant s \leqslant \tau_{-x}^{\downarrow}\right.$ ) (with an evident notation for $\tau_{-x}^{\downarrow}$ ) and the corresponding density has the form $\varphi\left(X_{\tau_{-x}^{\downarrow}}^{\downarrow}\right)$, where the function $\varphi$ is specified. 
Let us mention that we provide two other path decompositions that concern the excursion above the infimum (Theorem 4.6) and the process $\left(X_{s}^{\uparrow} ; 0 \leqslant s \leqslant \sigma_{x}^{\uparrow}\right.$ ) when $X_{\sigma_{x}^{\uparrow}}^{\uparrow}>x$ (Theorem 4.1).

\section{Preliminary results}

\subsection{Notation and basic assumptions}

In this section we state our notation and the assumptions made at different stages of the paper. We also recall fondamental results of fluctuation theory that are our starting-point and we give some simple facts concerning excursion theory applied to Lévy processes, that is the main tool we use.

We begin with some notations concerning the canonical space. Let $\Omega$ be the space of right-continuous functions with left limits from $(0,+\infty)$ to $\mathbb{R}$ (the so-called càdlàg functions space) endowed with the Skorokhod's topology. Let $\mathcal{F}$ stand for its Borel $\sigma$ algebra. For any path $\omega$ in $\Omega$ we define its lifetime $\zeta(\omega)$ by inf $\{t \geqslant 0: \omega(s)=\omega(t), \forall s \geqslant$ $t$, with the usual convention $\inf \emptyset=\infty$. For any time $t \geqslant 0$, we denote the jump of $\omega$ at $t$ by $\Delta \omega(t)=\omega(t)-\omega(t-)$; we also define the path respectively stopped at $t$, stopped just before $t$, reversed at $t$ and reversed just before $t$, by

$$
\begin{aligned}
& \omega(\cdot \wedge t)=(\omega(s \wedge t) ; s \geqslant 0), \quad \omega(\cdot \wedge t-)=\left(\omega(s \wedge t)-\Delta \omega(t) \mathbf{1}_{[t,+\infty)}(s) ; s \geqslant 0\right), \\
& \hat{\omega}^{t}=(\omega(t)-\omega((t-s)-) ; s \geqslant 0), \quad \hat{\omega}^{t-}=\hat{\omega}^{t}-\Delta \omega(t),
\end{aligned}
$$

with the convention $\omega(s-)=\omega(0)$ for any non-positive real number $s$. When $\zeta(\omega)$ is finite, $\hat{\omega}^{\zeta(\omega)}$ is well defined and simply denoted by $\hat{\omega}$. We use a non-standard notation for the shifted path at time $t$ defined by

$$
\omega \circ \theta_{t}=(\omega(s+t)-\omega(t) ; s \geqslant 0) .
$$

For any $x>0$, we denote by $\tau_{x}(\omega)$ and $\tau_{-x}(\omega)$ the first hitting time of respectively $(x,+\infty)$ and $(-\infty,-x)$ :

$$
\tau_{x}(\omega)=\inf \{s>0: \omega(s)>x\}, \quad \tau_{-x}(\omega)=\inf \{s>0: \omega(s)<-x\}
$$

(with the usual convention inf $\emptyset=+\infty$ ). For any time $t>0$, we also denote by $\sigma_{x}(t, \omega)$ and $\sigma_{-x}(t, \omega)$ the last passage time in respectively $(-\infty, x]$ and $[-x,+\infty)$ on the time interval $[0, t]$ :

$$
\sigma_{x}(t, \omega)=\sup \{0 \leqslant s \leqslant t: \omega(s) \leqslant x\}, \quad \sigma_{-x}(t, \omega)=\sup \{0 \leqslant s \leqslant t: \omega(s) \geqslant-x\}
$$

(with the convention $\sup \emptyset=+\infty$ ). We write $\sigma_{x}(\omega)=\lim _{t \rightarrow+\infty} \sigma_{x}(t, \omega)$, the limit being taken in $[0,+\infty]$. Next, we denote respectively by $\underline{g}_{t}(\omega)$ and $\bar{g}_{t}(\omega)$, the last infimum time and the last supremum time of $\omega$ before $t$ :

$$
\underline{g}_{t}(\omega)=\sup \left\{s \in[0, t): \inf _{[0, t]} \omega=\omega(s-) \wedge \omega(s)\right\}
$$


and

$$
\bar{g}_{t}(\omega)=\sup \left\{s \in[0, t): \sup _{[0, t]} \omega=\omega(s-) \vee \omega(s)\right\} .
$$

We also write $\underline{g}(\omega)=\lim _{t \rightarrow+\infty} \underline{g}_{t}(\omega)$ and $\bar{g}(\omega)=\lim _{t \rightarrow+\infty} \bar{g}_{t}(\omega)$ (note that these quantities may be infinite).

We denote by $X$ the canonical process on $\Omega: X_{t}(\omega)=\omega(t)$ and we consider the probability measure $\mathbf{P}$ on $(\Omega, \mathcal{F})$ under which $X$ is a Lévy process started at 0 , with characteristic exponent $\psi$ :

$$
\mathbf{E}\left[e^{i \lambda X_{t}}\right]=e^{-t \psi(\lambda)}, \quad t \geqslant 0, \lambda \in \mathbb{R} .
$$

By the Lévy-Khintchine theorem, $\psi$ has the form

$$
\psi(\lambda)=i a \lambda+b \lambda^{2}+\int \pi(d r)\left(1-e^{i \lambda r}+i \lambda r \mathbf{1}_{\{|r|<1\}}\right), \quad \lambda \in \mathbb{R},
$$

where $a$ is a real number, $b$ is non-negative and the Lévy measure $\pi$ is a Radon measure on $\mathbb{R}$ not charging 0 , which satisfies

$$
\int \pi(d r)\left(1 \wedge|r|^{2}\right)<+\infty
$$

If $J=\left\{s \geqslant 0: \Delta X_{s} \neq 0\right\}$, then the point measure $\mathcal{N}(d s d r)=\sum_{s \in J} \delta_{\left(s, \Delta X_{s}\right)}$ is a Poisson measure with intensity $d s \pi(d r)$.

Let us recall some path-properties of Lévy processes. For any $t \geqslant 0$, we have

$$
\widehat{X}^{t} \stackrel{(\text { law) }}{=}\left(X_{s} ; 0 \leqslant s \leqslant t\right)
$$

(see Bertoin [4]). This identity is refered to as the "duality property".

In the whole paper (Section 3 excepted), we make the following assumption:

Assumption (A). - The point 0 is regular for $(0,+\infty)$ and for $(-\infty, 0)$ with respect to $X$.

(In particular $X$ cannot be a subordinator or a compound Poisson process.) As a consequence of (A), we recall the following result (see Millar [15]): For any $t \geqslant 0$, the Lévy process $X$ reaches its infimum (respectively supremum) on $[0, t]$ at a unique instant that must be $\underline{g}_{t}(X)$ (respectively $\bar{g}_{t}(X)$ ).

For every $t \geqslant 0$, we write

$$
S_{t}=\sup _{s \in[0, t]} X_{s}, \quad I_{t}=\inf _{s \in[0, t]} X_{s} .
$$

It is well-known that $X-S$ and $X-I$ are strong Markov processes (see Bertoin [4], Chapter 6). Assumption (A) implies that 0 is regular for itself with respect to both these processes. Rogers has shown in [17] that this implies

$$
\mathbf{P}\left(\exists t \in(0,+\infty): X_{t-}=I_{t-}<X_{t}\right)=0
$$


and

$$
\mathbf{P}\left(\exists t \in(0,+\infty): X_{t-}=S_{t-}<S_{t}\right)=0 .
$$

Let us recall briefly the proof: we only need to show for any $\varepsilon>0$

$$
\mathbf{E}\left[\sum_{s \in J} \mathbf{1}_{\left\{X_{s-}=I_{s-}\right\}} \mathbf{1}_{(\varepsilon,+\infty)}\left(\Delta X_{s}\right)\right]=0 .
$$

Apply the compensation formula (see Bertoin [4], p. 7) to get

$$
\mathbf{E}\left[\sum_{s \in J} \mathbf{1}_{\left\{X_{s-}=I_{s-}\right\}} \mathbf{1}_{(\varepsilon,+\infty)}\left(\Delta X_{s}\right)\right]=\pi((\varepsilon,+\infty)) \int_{0}^{+\infty} d s \mathbf{P}\left(X_{s}=I_{s}\right) .
$$

But the duality property implies for any $s>0, \mathbf{P}\left(X_{s}=I_{s}\right)=\mathbf{P}\left(S_{s}=0\right)=0$, because 0 is regular for $(0,+\infty)$. A similar argument proves $(2)$.

We denote the local times of $X-I$ and $X-S$ at the level 0 by $\left(L_{t}\right)_{t \geqslant 0}$ and $\left(L_{t}^{*}\right)_{t \geqslant 0}$. They are uniquely determined up to a multiplicative constant specified in a forthcoming lemma. The limit in $[0,+\infty]$ of $L_{t}$ (respectively $L_{t}^{*}$ ) when $t$ goes to infinity is denoted by $L_{\infty}$ (respectively $L_{\infty}^{*}$ ). The quantity $L_{\infty}$ (respectively $L_{\infty}^{*}$ ) is a.s. finite or a.s. infinite according as $X$ drifts or not to $+\infty$ (respectively $-\infty$ ). If $L_{\infty}$ (respectively $L_{\infty}^{*}$ ) is finite a.s., then it is exponentially distributed with parameter denoted by $p$ (respectively $p^{*}$ ).

Eq. (1) and the dual result $\mathbf{P}\left(\exists t \in(0,+\infty): X_{t-}=S_{t-}>X_{t}\right)=0$ imply that $\mathbf{P}$ a.s. the sets $\left\{s \geqslant 0: X_{s}>I_{s}\right\}$ and $\left\{s \geqslant 0: X_{s}<S_{s}\right\}$ are open sets (we have denoted $\left(g_{i}, d_{i}\right), i \in \mathcal{I}$ and $\left(g_{j}, d_{j}\right), j \in \mathcal{I}^{*}$ their respective connected components). Let $\mathrm{m}$ denote the Lebesgue measure on $\mathbb{R}$. The duality property and Assumption (A) imply that $\mathrm{m}\left(s \geqslant 0: X_{s}=S_{s}\right)=\mathrm{m}\left(s \geqslant 0: X_{s}=I_{s}\right)=0$. Thus,

$$
\text { P-a.s. } \mathrm{m}\left(\mathbb{R} \backslash \bigcup_{i \in \mathcal{I}}\left(g_{i}, d_{i}\right)\right)=\mathrm{m}\left(\mathbb{R} \backslash \bigcup_{j \in \mathcal{I}^{*}}\left(g_{j}, d_{j}\right)\right)=0 .
$$

Let $N$ and $N^{*}$ be the excursion measures of $X$ above its infimum and under its supremum as defined in the first section. Observe that as soon as the Lévy measure $\pi$ charges $(-\infty, 0)$ (respectively $(0,+\infty)$ ), the set of excursions $\omega$ ending with a negative jump (respectively positive jump) has a positive $N$-measure (respectively $N^{*}$-measure). But thanks to (1) and the dual result, we see that excursions above the infimum and under the supremum leave 0 continuously.

Let $\left(L_{t}^{-1}\right)_{t \geqslant 0}$ and $\left(L_{t}^{*-1}\right)_{t \geqslant 0}$ be the right-continuous inverses of $L$ and $L^{*}$ :

$$
L_{t}^{-1}=\inf \left\{s \geqslant 0: L_{s}>t\right\}, \quad L_{t}^{*-1}=\inf \left\{s \geqslant 0: L_{s}^{*}>t\right\}
$$

(with the convention $\inf \emptyset=\infty$ ). Recall that $\mathbf{P}$-a.s.

$$
\bigcup_{s \geqslant 0}\left(L_{s-}^{-1}, L_{s}^{-1}\right)=\bigcup_{i \in \mathcal{I}}\left(g_{i}, d_{i}\right) \quad \text { and } \bigcup_{s \geqslant 0}\left(L_{s-}^{*-1}, L_{s}^{*-1}\right)=\bigcup_{j \in \mathcal{I}^{*}}\left(g_{j}, d_{j}\right) .
$$


For any $t \geqslant 0$ we define $U_{t}=-X_{L_{t}^{-1}}$ if $L_{\infty}>t$ and $U_{t}=+\infty$ if not. In a similar way, we define $U_{t}^{*}=X_{L_{t}^{*-1}}$ if $L_{\infty}^{*}>t$ and $U_{t}^{*}=+\infty$ if not. The processes $\left(L^{-1}, U\right)$ and $\left(L^{*-1}, U^{*}\right)$ are called the ladder processes. They are two-dimensional subordinators killed at respective rates $p$ and $p^{*}$; their bivariate Laplace exponents are denoted by

$$
\begin{aligned}
& \kappa(\alpha, \beta)=-\log \mathbf{E}\left[\exp \left(-\alpha L_{1}^{-1}-\beta U_{1}\right)\right] \text { and } \\
& \kappa^{*}(\alpha, \beta)=-\log \mathbf{E}\left[\exp \left(-\alpha L_{1}^{*-1}-\beta U_{1}^{*}\right)\right]
\end{aligned}
$$

(see Bertoin [4], Chapter 6 for a detailed account). Next, we define the two potential measures $\mathcal{U}$ and $\mathcal{U}^{*}$ associated with $U$ and $U^{*}$ :

$$
\left\{\begin{array}{l}
\int_{\mathbb{R}} \mathcal{U}(d x) f(x)=\mathbf{E}\left[\int_{0}^{L_{\infty}} d v f\left(U_{v}\right)\right] \\
\int_{\mathbb{R}} \mathcal{U}^{*}(d x) f(x)=\mathbf{E}\left[\int_{0}^{L_{\infty}^{*}} d u f\left(U_{u}^{*}\right)\right] .
\end{array}\right.
$$

Let $d^{*}$ be the drift coefficient of the subordinator $U^{*}: d^{*}=\lim _{\beta \rightarrow+\infty} \kappa^{*}(0, \beta) / \beta$. We recall the following result, due to Kesten [11] (see also Bertoin [4], Chapter 3, Theorem 5): assume that $d^{*}$ is positive, and let $u^{*}:(-\infty,+\infty) \longrightarrow[0,+\infty)$ be the coexcessive version of the density of $\mathcal{U}^{*}$. Then $u^{*}$ is continuous and positive on $(0,+\infty)$, $u^{*}\left(0^{+}\right)=1 / d^{*}$, and

$$
\mathbf{P}\left(X_{\tau_{x}}=x\right)=d^{*} u^{*}(x), \quad x>0,
$$

where for convenience we write $\tau_{x}$ instead of $\tau_{x}(X)$. We prove the following simple lemma that will be used in Section 4.

LEMMA 2.1. - Assume (A) and suppose that $d^{*}$ is positive. Then for any nonnegative measurable functional $F$ on $\Omega$,

$$
\mathbf{E}\left[\int_{0}^{L_{\infty}^{*}} d u F\left(X_{\cdot \wedge L_{u}^{*-1}}\right)\right]=\int_{0}^{+\infty} d x u^{*}(x) \mathbf{E}\left[F\left(X_{\cdot \wedge \tau_{x}}\right) \mid X_{\tau_{x}}=x\right] .
$$

Proof. - Set $A=\int_{0}^{+\infty} d x \mathbf{E}\left[F\left(X_{\cdot \wedge \tau_{x}}\right) ; X_{\tau_{x}}=x\right]$ and for any positive number $x$ define $H_{x}=L_{\tau_{x}}^{*}$. Thanks to (A), we check path by path that

$$
\text { P-a.s. on } \quad\left\{\tau_{x}<\infty\right\}, \quad L_{H_{x}}^{*-1}=\tau_{x} .
$$

Thus

$$
A=\int_{0}^{+\infty} d x \mathbf{E}\left[F\left(X_{\left.\cdot \wedge L_{H_{x}}^{*-1}\right)} ; X_{\tau_{x}}=x\right] .\right.
$$

Denote by $C$ the random set $\left\{x \geqslant 0: X_{\tau_{x}}=x\right\}$ and define the measures $\mu$ and $\nu$ by

$$
\left\{\begin{array}{l}
\mu(d x)=\mathbf{1}_{C}(x) \mathrm{m}(d x), \\
\int \nu(d u) f(u)=\int \mu(d x) f\left(H_{x}\right) .
\end{array}\right.
$$


Then,

$$
A=\mathbf{E}\left[\int_{\left[0, S_{\infty}\right)} \mu(d x) F\left(X_{\cdot \wedge L_{H_{x}}^{*-1}}\right)\right]=\mathbf{E}\left[\int_{\left[0, L_{\infty}^{*}\right)} v(d u) F\left(X_{\cdot \wedge L_{u}^{*-1}}\right)\right] .
$$

For any positive number $a$,

$$
\begin{aligned}
v([0, a]) & =\mathrm{m}\left(\left\{x \geqslant 0: X_{\tau_{x}}=x ; H_{x} \leqslant a\right\}\right) \\
& =\mathrm{m}\left(\left\{x \geqslant 0: X_{\tau_{x}}=x ; \tau_{x} \leqslant L_{a}^{*-1}\right\}\right)=\mathrm{m}\left(C \cap\left[0, U_{a}^{*}\right]\right) .
\end{aligned}
$$

Let us first consider the case $U_{a}^{*}<\infty$ : If there exists some $s$ in $[0, a]$ such that $x \in\left(U_{s-}^{*}, U_{s}^{*}\right)$, then, $\tau_{x}=L_{s}^{*-1}$ and $U_{s}^{*}=X_{\tau_{x}}>x$. Thus,

$$
\bigcup_{0 \leqslant s \leqslant a}\left(U_{s-}^{*}, U_{s}^{*}\right) \subset C^{c} \cap\left[0, U_{a}^{*}\right] .
$$

Let $x$ be in $C^{c} \cap\left[0, U_{a}^{*}\right]$. Then, $X_{\tau_{x}}>x$. By (2), it follows that $\tau_{x}$ must be the end-point of some excursion interval of $S-X$ above 0 that is included in $\left[0, L_{a}^{*-1}\right]$. Then, by (4) there exists some $s$ in $[0, a]$ such that

$$
L_{s-}^{*-1}<L_{s}^{*-1}=\tau_{x} \quad \text { and } \quad S_{\tau_{x}-}=U_{s-}^{*} \leqslant x<U_{s}^{*}=X_{\tau_{x}} .
$$

Hence,

$$
C^{c} \cap\left[0, U_{a}^{*}\right] \subset \bigcup_{0 \leqslant s \leqslant a}\left[U_{s-}^{*}, U_{s}^{*}\right) .
$$

By combining this with the previous inclusion we get $\mathrm{m}\left(C^{c} \cap\left[0, U_{a}^{*}\right]\right)=\sum_{0 \leqslant s \leqslant a} \Delta U_{s}^{*}$. But the Lévy-Itô representation of $U^{*}$ guarantees that $\mathbf{P}$-a.s.

$$
U_{a}^{*}=d^{*} a+\sum_{0 \leqslant s \leqslant a} \Delta U_{s}^{*}, \quad 0 \leqslant a<L_{\infty}^{*} .
$$

Then, $\mathbf{P}$-a.s. for every $a$ in $\left[0, L_{\infty}^{*}\right), v([0, a])=d^{*} a$. Next, observe that for any $a>L_{\infty}^{*}$, $v([0, a])=\mathrm{m}(C)=d^{*} L_{\infty}^{*}$. Thus, $\mathbf{P}$-a.s.

$$
v(d x)=d^{*} \mathbf{1}_{\left[0, L_{\infty}^{*}\right)}(x) m(d x) .
$$

The desired result follows from (5) and the identity $\mathbf{P}\left(X_{\tau_{x}}=x\right)=d^{*} u^{*}(x)$.

Let us introduce some notations: for any positive time $t$ and any path $\omega$, we denote the pre-infimum and the post-infimum path on the interval $[0, t]$ by:

$$
\left\{\begin{array}{l}
\stackrel{\omega^{t}}{\leftarrow}=\omega\left(\cdot \wedge \underline{g}_{t}(\omega)\right), \\
\stackrel{\omega}{\rightarrow}=\left(\omega \circ \underline{\theta}_{\underline{g}_{t}}(\omega)\right)\left(\cdot \wedge\left(t-\underline{g}_{t}(\omega)\right)\right) .
\end{array}\right.
$$

We also denote the pre-supremum and post-supremum processes on $[0, t]$ by $\overleftarrow{\omega}^{t}$ and $\vec{\omega}^{t}$ :

$$
\left\{\begin{array}{l}
\overleftarrow{\omega}^{t}=\omega\left(\cdot \wedge \bar{g}_{t}(\omega)\right) \\
\vec{\omega}^{t}=\left(\omega \circ \theta \bar{g}_{t}(\omega)\right)\left(\cdot \wedge\left(t-\bar{g}_{t}(\omega)\right)\right)
\end{array}\right.
$$


We often use the following lemma in Section 4:

Lemma 2.2. - Assume (A). Let $T$ be independent of $X$ and exponentially distributed with parameter $\alpha>0$. Then, ${\underset{X}{\leftarrow}}^{T}$ and $\underset{\rightarrow}{\rightarrow}$ are mutually independent and the following identities hold for any nonnegative measurable functional $F$ on $\Omega$ :

(i) $\mathbf{E}\left[F\left({\underset{X}{\leftarrow}}^{T}\right)\right]=\kappa(\alpha, 0) \mathbf{E}\left[\int_{0}^{L \infty} d v e^{-\alpha L_{v}^{-1}} F\left(X_{\cdot \wedge L_{v}^{-1}}\right)\right]$.

(ii) $\mathbf{E}\left[F\left(\stackrel{X}{\rightarrow}^{T}\right)\right]=\frac{1}{\kappa(\alpha, 0)} N\left(\int_{0}^{\zeta} d s \alpha e^{-\alpha s} F(\omega \cdot \wedge s)\right)$.

Proof. - Let $G$ be any nonnegative measurable functional on $\Omega$. We have

$$
\mathbf{E}\left[F\left({\underset{X}{\leftarrow}}^{T}\right) G\left(\underline{X}^{T}\right)\right]=\int_{0}^{\infty} d t \alpha e^{-\alpha t} \mathbf{E}\left[F\left({\underset{X}{\leftarrow}}^{t}\right) G\left(\underline{X}^{t}\right)\right] .
$$

By (3) and by the definition of the excursions above the infimum, we have $\mathbf{P}$-a.s.

$$
\int_{0}^{\infty} d t \alpha e^{-\alpha t} F\left({\underset{X}{\leftarrow}}^{t}\right) G\left({\underset{X}{X}}^{t}\right)=\sum_{i \in \mathcal{I}} e^{-\alpha g_{i}} F\left(X \cdot \wedge g_{i}\right) \int_{0}^{\zeta_{i}} d s \alpha e^{-\alpha s} G\left(\omega^{i}(\cdot \wedge s)\right) .
$$

Apply the compensation formula to get

$$
\mathbf{E}\left[F\left({\underset{X}{\leftarrow}}^{T}\right) G\left(\underline{X}^{T}\right)\right]=\mathbf{E}\left[\int_{0}^{L_{\infty}} d v e^{-\alpha L_{v}^{-1}} F\left(X_{\cdot \wedge L_{v}^{-1}}\right)\right] N\left(\int_{0}^{\zeta} d s \alpha e^{-\alpha s} G\left(\omega_{\cdot \wedge s}\right)\right)
$$

and the following identities yield (i) and (ii):

$$
N\left(\int_{0}^{\zeta} d s \alpha e^{-\alpha s}\right)=N\left(1-e^{-\alpha \zeta}\right)=\kappa(\alpha, 0)
$$

and

$$
\mathbf{E}\left[\int_{0}^{L_{\infty}} d v e^{-\alpha L_{v}^{-1}}\right]=\frac{1}{\kappa(\alpha, 0)}
$$

We now specify the normalization of $L$ and $L^{*}$ thanks to the following proposition.

Proposition 2.3. - Assume (A). Fix the normalization of $L$. Then, the normalization of $L^{*}$ can be chosen in order to have for any nonnegative measurable functional $F$ on $\Omega$

$$
N\left(\int_{0}^{\zeta} d s F\left(\hat{\omega}^{s}\right)\right)=\mathbf{E}\left[\int_{0}^{L_{\infty}^{*}} d u F\left(X_{\cdot \wedge L_{u}^{*-1}}\right)\right]
$$


and the dual identity

$$
N^{*}\left(\int_{0}^{\zeta} d s F\left(\hat{\omega}^{s}\right)\right)=\mathbf{E}\left[\int_{0}^{L_{\infty}} d v F\left(X_{\cdot \wedge L_{v}^{-1}}\right)\right] .
$$

Proof. - We denote by $\underset{\leftarrow}{\stackrel{X^{T}}{\leftarrow}}$ the path $\underset{\leftarrow}{\underline{X}}$ reversed at its lifetime $\underline{g}_{T}$. Observe that

$$
\widehat{X}^{T}=\widehat{X}^{T} \circ \theta_{\bar{g}_{T}\left(\widehat{X}^{T}\right)}
$$

(we use the fact that the minimum of $X$ over $[0, T]$ is attained $\mathbf{P}$-a.s. at a unique time). We also denote by $\widehat{X}^{T}$ the path $\underset{\rightarrow}{\rightarrow}$ reversed at its lifetime $T-\underline{g}_{T}$. Similarly, we see that

$$
{\widehat{X^{T}}}^{T}=\widehat{X}_{\cdot \wedge \bar{g}_{T}\left(\widehat{X}^{T}\right)} \cdot
$$

The duality property implies that

$$
\left({\stackrel{\left(X^{T}\right.}{\leftarrow}}_{\underline{X}^{T}}^{\rightarrow}\right) \stackrel{(\text { law })}{=}\left(\vec{X}^{T}, \overleftarrow{X}^{T}\right)
$$

Let $G$ be any nonnegative measurable functional on $\Omega$. Use Lemma 2.2 to get:

$$
\mathbf{E}\left[F\left(\widehat{\underline{X}}^{T}\right) G\left(\widehat{X}^{T}\right)\right]=\mathbf{E}\left[\int_{0}^{L_{\infty}} d v e^{-\alpha L_{v}^{-1}} F\left(\widehat{X}^{L_{v}^{-1}}\right)\right] N\left(\int_{0}^{\zeta} d s \alpha e^{-\alpha s} G\left(\hat{\omega}^{s}\right)\right) .
$$

On the other hand, by replacing $X$ with $-X$, we see that Lemma 2.2 also implies

$$
\mathbf{E}\left[F\left(\vec{X}^{T}\right) G\left(\overleftarrow{X}^{T}\right)\right]=\mathbf{E}\left[\int_{0}^{L_{\infty}^{*}} d u e^{-\alpha L_{u}^{*-1}} G\left(X_{\cdot \wedge L_{u}^{*-1}}\right)\right] N^{*}\left(\int_{0}^{\zeta} d s \alpha e^{-\alpha s} F\left(\omega_{\cdot \wedge s}\right)\right)
$$

Thus, for any $\alpha>0$

$$
\begin{array}{r}
\mathbf{E}\left[\int_{0}^{L_{\infty}^{*}} d u e^{-\alpha L_{u}^{*-1}} G\left(X_{\cdot \wedge L_{u}^{*-1}}\right)\right] N^{*}\left(\int_{0}^{\zeta} d s e^{-\alpha s} F\left(\omega_{\cdot \wedge s}\right)\right) \\
=\mathbf{E}\left[\int_{0}^{L_{\infty}} d v e^{-\alpha L_{v}^{-1}} F\left(\widehat{X}^{L_{v}^{-1}}\right)\right] N\left(\int_{0}^{\zeta} d s e^{-\alpha s} G\left(\hat{\omega}^{s}\right)\right) .
\end{array}
$$

By letting $\alpha$ go to 0 , we see that the ratio

$$
\frac{N^{*}\left(\int_{0}^{\zeta} d s F\left(\omega_{\cdot \wedge s}\right)\right)}{\mathbf{E}\left[\int_{0}^{L_{\infty}} d v F\left(\widehat{X}^{L_{v}^{-1}}\right)\right]}
$$


does not depend on $F$, provided it is well-defined (that is the denominator is positive and finite). Furthermore this ratio coincides with

$$
\frac{N\left(\int_{0}^{\zeta} d s G\left(\widehat{\omega}^{s}\right)\right)}{\mathbf{E}\left[\int_{0}^{L_{\infty}^{*}} d u G\left(X_{\cdot \wedge L_{u}^{*-1}}\right)\right]}
$$

for any $G$ such that the denominator is positive and finite. We can choose the normalization of $N^{*}$, or equivalently $L^{*}$, so that both ratios are equal to 1 .

In the spectrally positive case, the first identity of Proposition 2.3 has been proved by Le Gall and Le Jan in [12] by a different method.

Immediate applications of Proposition 2.3 are the following identities due to Silverstein (see [18]), also mentioned in Rogers' paper [17]:

$$
\left\{\begin{array}{l}
N\left(\int_{0}^{\zeta} d s e^{-\alpha s-\beta \omega_{s}}\right)=1 / \kappa^{*}(\alpha, \beta), \\
N^{*}\left(\int_{0}^{\zeta} d s e^{-\alpha s+\beta \omega_{s}}\right)=1 / \kappa(\alpha, \beta) .
\end{array}\right.
$$

We can also derive from Proposition 2.3 the Wiener-Hopf factorisation of the ladder exponents:

$$
\kappa(\alpha, i \beta) \kappa^{*}(\alpha,-i \beta)=\alpha+\psi(\beta) .
$$

Indeed, (3) gives the following decomposition:

$$
\int_{0}^{+\infty} d t e^{-\alpha t+i \beta X_{t}}=\sum_{i \in \mathcal{I}} \int_{g_{i}}^{d_{i}} d t e^{-\alpha t+i \beta X_{t}}=\sum_{i \in \mathcal{I}} e^{-\alpha g_{i}+i \beta X_{g_{i}}} \int_{0}^{\zeta_{i}} d s e^{-\alpha s+i \beta \omega_{i}(s)} .
$$

Taking the expectations and using the compensation formula, we get

$$
1 /(\alpha+\psi(\beta))=\mathbf{E}\left[\int_{0}^{L_{\infty}} d u e^{-\alpha L_{u}^{-1}+i \beta X_{L_{u}^{-1}}}\right] N\left(\int_{0}^{\zeta} d s e^{-\alpha s+i \beta \omega_{s}}\right),
$$

which yields the Wiener-Hopf factorization thanks to Proposition 2.3.

\subsection{The Lévy process conditioned to stay positive or negative}

We introduce now the process conditioned to stay positive, respectively negative, denoted by $X^{\uparrow}$, respectively $X^{\downarrow}$. Bertoin in [3] provides a pathwise construction of $X^{\uparrow}$ and $X^{\downarrow}$ from concatenation of the excursions of $X$ in $(0,+\infty)$, respectively $(-\infty, 0)$. Let us recall briefly this construction whose details can be found in [3], Section 3.

Although Bertoin's construction holds in a general setting, we assume (A). We denote by $\left(\mathcal{F}_{t}\right)_{t \geqslant 0}$ the natural filtration of $X$ completed with the $\mathbf{P}$-null sets of $\mathcal{F}$. Then, $X$ is a semimartingale. Its continuous local martingale part is proportional to a standard Brownian motion and is independent of the non-continuous part. Let us denote by $\ell$ its 
semimartingale local time at 0 . We consider

$$
A_{t}^{+}=\int_{0}^{t} d s \mathbf{1}_{\left\{X_{s}>0\right\}} \quad \text { and } \quad A_{t}^{-}=\int_{0}^{t} d s \mathbf{1}_{\left\{X_{s} \leqslant 0\right\}} .
$$

Let us denote by $\alpha^{+}$, respectively $\alpha^{-}$, the right-continuous inverse of $A^{+}$, respectively $A^{-}$:

$$
\alpha_{t}^{+}=\inf \left\{s \geqslant 0: A_{s}^{+}>t\right\} \quad \text { and } \quad \alpha_{t}^{-}=\inf \left\{s \geqslant 0: A_{s}^{-}>t\right\}
$$

(with the usual convention $\inf \emptyset=\infty$ ). Let $x$ be a real number. We denote its positive part, respectively negative part, by $x_{+}$, respectively $x_{-}$. We define a new process $X^{\uparrow}$ by

$$
X_{t}^{\uparrow}=X_{\alpha_{t}^{+}}+\frac{1}{2} \ell_{\alpha_{t}^{+}}+\sum_{0<s \leqslant \alpha_{t}^{+}} \mathbf{1}_{\left\{X_{s} \leqslant 0\right\}}\left(X_{s-}\right)_{+}+\mathbf{1}_{\left\{X_{s}>0\right\}}\left(X_{s-}\right)_{-} \quad \text { if } t<A_{\infty}^{+}
$$

and by $X_{t}^{\uparrow}=+\infty$ if not. When $X$ has no Brownian part, $X^{\uparrow}$ can be viewed as the concatenation of the excursions of $X$ in $(0,+\infty)$. Similarly, we define $X^{\downarrow}$ by

$$
X_{t}^{\downarrow}=X_{\alpha_{t}^{-}}-\frac{1}{2} \ell_{\alpha_{t}^{-}}-\sum_{0<s \leqslant \alpha_{t}^{-}} \mathbf{1}_{\left\{X_{s} \leqslant 0\right\}}\left(X_{s-}\right)_{+}+\mathbf{1}_{\left\{X_{s}>0\right\}}\left(X_{s-}\right)_{-} \quad \text { if } t<A_{\infty}^{-}
$$

and by $X_{t}^{\downarrow}=-\infty$ if not. The laws of $X^{\uparrow}$ and $X^{\downarrow}$ can be recovered by a harmonic transform: Denote by $q_{t}^{+}(x, d y)$ and $q_{t}^{-}(x, d y)$ the semigroup of the Lévy process killed respectively in $(-\infty, 0]$ and $[0,+\infty)$. One can show (see Silverstein in [18]) that the functions $\mathcal{U}^{*}([0, x])$ and $\mathcal{U}([0, x])$ are superharmonic respectively for $q^{+}$and for $q^{-}$ and that the following kernels

$$
p_{t}^{+}(x, d y)=\frac{\mathcal{U}([0, y])}{\mathcal{U}([0, x]} q_{t}^{+}(x, d y), \quad x>0,
$$

and

$$
p_{t}^{-}(x, d y)=\frac{\mathcal{U}^{*}([0,-y])}{\mathcal{U}^{*}([0,-x])} q_{t}^{-}(x, d y), \quad x<0,
$$

define two sub-markovian semigroups. Bertoin has shown in [3], Theorem 3.4 that $X^{\uparrow}$ and $X^{\downarrow}$ are Markov processes started at 0 with respective semigroups $p^{+}$and $p^{-}$. If $X$ does not drift to $-\infty$, respectively $+\infty$, then, $p^{+}$, respectively $p^{-}$, is markovian and $X^{\uparrow}$, respectively $X^{\downarrow}$, has an infinite lifetime. More precisely, if $X$ does not drift to $-\infty$, then, we can show that

$$
X_{t}^{\uparrow}<\infty, \quad t \geqslant 0 \quad \text { and } \quad \lim _{t \rightarrow \infty} X_{t}^{\uparrow}=\infty .
$$

Proof. - If $X$ does not drift to $-\infty$, it is easy to check that $\lim _{t \rightarrow \infty} A_{t}^{+}=\infty, \mathbf{P}$-a.s. and then, $X_{t}^{\uparrow}<\infty, t>0$. If $X$ drifts to $+\infty$, we have $\alpha_{t+A_{\sigma_{0}}^{+}}^{+}=\sigma_{0}+t ; t \geqslant 0$, where 
$\sigma_{0}=\sup \left\{s \geqslant 0: X_{s} \leqslant 0\right\}<\infty$. Thus,

$$
X_{t+A_{\sigma_{0}}^{+}}^{\uparrow} \geqslant X_{\sigma_{0}+t} \underset{t \rightarrow \infty}{\longrightarrow}+\infty .
$$

If $X$ oscillates, we must consider to cases: Suppose first that $\pi \neq 0$, then,

$$
\lim _{t \rightarrow \infty} X_{t}^{\uparrow} \geqslant \sum_{s>0} \mathbf{1}_{\left\{X_{s} \leqslant 0\right\}}\left(X_{s-}\right)_{+}+\mathbf{1}_{\left\{X_{s}>0\right\}}\left(X_{s-}\right)_{-}=\infty .
$$

If $\pi$ is null, then by assumption (A) there is a Brownian component and $\lim _{t \rightarrow \infty} \ell_{t}=\infty$ that yields the desired result.

In particular cases, we recover "classical" definitions of the process conditioned to stay positive:

- In the Brownian case, $\mathcal{U}^{*}([0, x])=\mathcal{U}((-x, 0])=x$ and $p^{+}$is the semigroup of the three-dimensional Bessel process started at 0 .

- In the spectrally positive case and the stable case, Chaumont has shown in [7] and [9] that if the Lévy process does not drift to $-\infty$ and if 0 is regular for $(0,+\infty)$, then, for any bounded $\mathcal{F}_{t}$ measurable functional $F$ that is continuous for the Skorokhod topology on $\Omega$ :

$$
\mathbf{E}\left[F\left(X^{\uparrow}\right)\right]=\lim _{x \rightarrow 0} \lim _{T \rightarrow+\infty} \mathbf{E}_{x}\left[F(X) \mid I_{T} \geqslant 0\right] .
$$

- In the spectrally negative case, Bertoin (see [3]) gives another construction of $X^{\uparrow}$ that generalizes Pitman's theorem for Brownian motion (see Pitman [16]).

Let us denote by $\widehat{X}^{t}$ the path ${\underset{X}{t}}^{t}$ reversed at its lifetime $\underline{g}_{t}$. We denote by $\left(X_{s}^{\downarrow} ; 0 \leqslant\right.$ $s<A_{t}^{-}$) (respectively $\left(X_{s}^{\uparrow} ; 0 \leqslant s<A_{t}^{+}\right)$) the process $X^{\downarrow}$ (respectively $X^{\uparrow}$ ) stopped at the random time $A_{t}^{-}$(respectively $A_{t}^{+}$). We need the following theorem due to Bertoin that links the process conditioned to stay positive with excursion theory:

THEOREM 2.4 (Bertoin [3], Theorem 3.1). - For every $t>0$, the following identity holds

$$
\left(\widehat{X}^{t}, \stackrel{X}{X}^{t}\right) \stackrel{(\text { law) }}{=}\left(\left(X_{s}^{\downarrow}\right)_{0 \leqslant s<A_{t}^{-}},\left(X_{s}^{\uparrow}\right)_{0 \leqslant s<A_{t}^{+}}\right) .
$$

Remark. - If $X$ drifts to $+\infty$, then, the previous identity holds with $t=\infty$ as to say that $X^{\uparrow}$ has the same law as the post-infimum process (see Millar in [14]).

In Section 4, we use another identity that is proved in [1] (see also [13]). From now on until the end of this section, we assume that $X$ does not drift to $-\infty$. For any $t>0$ we set

$$
J_{t}=\inf _{s \in[t, \infty)} X_{s}^{\uparrow}
$$

and

$$
\bar{d}_{t}=\inf \left\{s>t: S_{s}=X_{s}\right\}
$$


LEMMA 2.5 (Bertoin [1], Lemme 4).-

$$
\left(S_{\left(\bar{d}_{t}+\bar{g}_{t}-t\right)-}-X_{\left(\bar{d}_{t}+\bar{g}_{t}-t\right)-}, S_{\bar{d}_{t}}\right)_{t \geqslant 0} \stackrel{\text { (law) }}{=}\left(X_{t}^{\uparrow}-J_{t}, J_{t}\right)_{t \geqslant 0} .
$$

Proof. - Although Bertoin in Lemma 4 of [1] only considers the case of $X \rightarrow+\infty$ (taking $X^{\uparrow}$ as the post-infimum process), the proof can be adapted when $X$ oscillates thanks to Theorem 2.4 and the arguments are exactly the same.

The process $X^{\uparrow}-J$ is a strong Markov process and 0 is a regular value. We denote by $K$ its local time at 0 normalized in order it is distributed as $L^{*}$. Let us denote by $\left(g_{i}, d_{i}\right)$, $i \in \mathcal{I}^{\uparrow}$ the excursion intervals of $X^{\uparrow}-J$ above 0 :

$$
\left\{s \geqslant 0: X_{s}^{\uparrow}>J_{s}\right\}=\bigcup_{i \in \mathcal{I}^{\uparrow}}\left(g_{i}, d_{i}\right) .
$$

We define

$$
w^{i}(s)=\Delta J_{g_{i}}+\left(X^{\uparrow}-J\right)_{\left(s+g_{i}\right) \wedge d_{i}}, \quad s \geqslant 0, i \in \mathcal{I}^{\uparrow} .
$$

Then, Lemma 2.5 implies that

$$
\mathcal{N}^{\uparrow}(d k d w)=\sum_{i \in \mathcal{I} \uparrow} \delta_{\left(K_{g_{i}}, w^{i}\right)}
$$

is a Poisson point process with intensity $d k \widehat{N}^{*}(d w)$, where $\widehat{N}^{*}$ is the law of $\widehat{\omega}^{\zeta}$ under $N^{*}(d \omega)$. We use this result in Section 4.

\section{Reversion formulas}

Let $x$ and $t$ be two positive real numbers. We first decompose the law of $\widehat{X}^{\sigma_{x}(t)}$ on the event $\left\{X_{\sigma_{x}(t)}>x\right\}$ in terms of the law of $X$, the Lévy measure $\pi$ and the function $\Lambda$ that is defined on $(0,+\infty) \times(0,+\infty)$ by $\Lambda(s, a)=\mathbf{P}\left(I_{s} \geqslant-a\right)$. From classical fluctuation identities we have

$$
\int_{(0,+\infty) \times(0,+\infty)} d s d a \exp (-\lambda s-\mu a) \Lambda(s, a)=\frac{\kappa(\lambda, 0)}{\lambda \mu \kappa(\lambda, \mu)} .
$$

We also write $\Lambda(a)$ for the limit $\lim _{s \rightarrow+\infty} \Lambda(s, a)$ that is positive if and only if $X$ drifts to $+\infty$ (or equivalently $p>0$ ). To simplify notations, we write $\mathbf{P}_{r}$ for the law of the Lévy process started at $r$. We prove the first reversion formula:

THEOREM 3.1. - Assume that $\pi$ charges $(0,+\infty)$. Then for any positive numbers $x$ and $t$, and for any bounded measurable functional $F$ on $\Omega$,

$$
\begin{aligned}
\mathbf{E} & {\left[F\left(\widehat{X}^{\sigma_{x}(t)}\right) ; \sigma_{x}(t)<\infty ; X_{\sigma_{x}(t)}>x\right] } \\
& =\int_{(0,+\infty)} \pi(d r) \int_{0}^{t} d u \mathbf{E}_{r}\left[F\left(X_{\cdot \wedge u}\right) \Lambda\left(t-u, X_{u}-x\right) ; x<X_{u} \leqslant x+r\right] .
\end{aligned}
$$


Consequences. (i) If $X$ drifts to $+\infty$, then $\Lambda(a)>0$ for any positive real number $a$. Thanks to Theorem 3.1 we get

$$
\begin{aligned}
\mathbf{E} & {\left[F\left(\hat{X}^{\sigma_{x}(t)}\right) \frac{\Lambda\left(X_{\sigma_{x}(t)}-x\right)}{\Lambda\left(t-\sigma_{x}(t), X_{\sigma_{x}(t)}-x\right)} ; \sigma_{x}(t)<\infty ; X_{\sigma_{x}(t)}>x\right] } \\
& =\int_{(0,+\infty)} \pi(d r) \int_{0}^{t} d u \mathbf{E}_{r}\left[F\left(X_{\cdot \wedge u}\right) \Lambda\left(X_{u}-x\right) ; x<X_{u} \leqslant x+r\right] .
\end{aligned}
$$

Observe that $\mathbf{P}$-a.s. $\sigma_{x}(t)=\sigma_{x}$ for all $t$ sufficiently large. Thus, $\mathbf{P}$-a.s.

$$
F\left(\widehat{X}^{\sigma_{x}(t)}\right) \frac{\Lambda\left(X_{\sigma_{x}(t)}-x\right)}{\Lambda\left(t-\sigma_{x}(t), X_{\sigma_{x}(t)}-x\right)} \mathbf{1}_{\left\{\sigma_{x}(t)<\infty ; X_{\left.\sigma_{x}(t)>x\right\}}\right.} \underset{t \rightarrow \infty}{\longrightarrow} F\left(\widehat{X}^{\sigma_{x}}\right) \mathbf{1}_{\left\{X_{\sigma_{x}}>x\right\}} .
$$

Since $\Lambda\left(X_{\sigma_{x}(t)}-x\right) / \Lambda\left(t-\sigma_{x}(t), X_{\sigma_{x}(t)}-x\right)$ is smaller than 1 , dominated convergence applies and we deduce from (9) that

$$
\begin{aligned}
\mathbf{E} & {\left[F\left(\widehat{X}^{\sigma_{x}}\right) ; X_{\sigma_{x}}>x\right] } \\
& =\int_{(0,+\infty)} \pi(d r) \int_{0}^{+\infty} d u \mathbf{E}_{r}\left[F\left(X_{\cdot \wedge u}\right) \Lambda\left(X_{u}-x\right) ; x<X_{u} \leqslant x+r\right] .
\end{aligned}
$$

(ii) By the duality property applied in the right side of Theorem 3.1, we see that under $\mathbf{P}\left(\cdot \mid X_{\sigma_{x}(t)}>x\right)$

$$
X_{\cdot \wedge \sigma_{x}(t)-} \stackrel{(\text { law })}{=} \widehat{X}^{\sigma_{x}(t)-} .
$$

Proof of Theorem 3.1. - Let $\varepsilon$ be a positive real number and let $\left(\sigma_{n}\right)_{n \geqslant 0}$ be the increasing sequence of the jump times $\left\{s \geqslant 0: \Delta X_{s}>\varepsilon\right\}$. Recall that $\sum_{s \in J} \delta_{\left(s, \Delta X_{s}\right)}$ is a Poisson measure with intensity $d l \pi(d r)$. Let $f$ be a bounded function on $\mathbb{R}$. Consider the event $A_{\varepsilon}=\left\{\sigma_{x}(t)<\infty ; \inf _{s \in\left[\sigma_{x}(t), t\right]} X_{s}>\varepsilon+x\right\}$ and set

$$
a(\varepsilon)=\mathbf{E}\left[F\left(\widehat{X}^{\sigma_{x}(t)-}\right) f\left(\Delta X_{\sigma_{x}(t)}\right) ; A_{\varepsilon}\right] .
$$

Observe that

$$
a(\varepsilon)=\sum_{n \geqslant 0} \mathbf{E}\left[F\left(\widehat{X}^{\sigma_{n}-}\right) f\left(\Delta X_{\sigma_{n}}\right) ; \sigma_{n}<t ; X_{\sigma_{n}-}<x ; X_{\sigma_{n}}+\inf _{\left[0, t-\sigma_{n}\right]} X \circ \theta_{\sigma_{n}}>\varepsilon+x\right] .
$$

Apply the Markov property at $\sigma_{n}$ in order to get

$$
a(\varepsilon)=\sum_{n \geqslant 0} \mathbf{E}\left[F\left(\widehat{X}^{\sigma_{n}-}\right) f\left(\Delta X_{\sigma_{n}}\right) \Lambda\left(t-\sigma_{n}, X_{\sigma_{n}}-\varepsilon-x\right) ; \sigma_{n}<t ; X_{\sigma_{n}-}<x\right] .
$$

Then,

$$
a(\varepsilon)=\mathbf{E}\left[\sum_{s \in J: s \leqslant t} \mathbf{1}_{\left\{\Delta X_{s}>\varepsilon ; X_{s-}<x\right\}} F\left(\widehat{X}^{s-}\right) f\left(\Delta X_{s}\right) \Lambda\left(t-s, X_{s-}+\Delta X_{s}-\varepsilon-x\right)\right] .
$$


Apply the compensation formula to get:

$$
a(\varepsilon)=\int_{(\varepsilon,+\infty)} \pi(d r) f(r) \int_{0}^{t} d u \mathbf{E}\left[F\left(\widehat{X}^{u}\right) \Lambda\left(t-u, X_{u}+r-\varepsilon-x\right) ; x+\varepsilon-r<X_{u}<x\right]
$$

and by duality

$$
a(\varepsilon)=\int_{(\varepsilon,+\infty)} \pi(d r) f(r) \int_{0}^{t} d u \mathbf{E}\left[F\left(X_{\cdot \wedge u}\right) \Lambda\left(t-u, X_{u}+r-\varepsilon-x\right) ; x+\varepsilon-r<X_{u}<x\right] .
$$

Next, observe that $\mathbf{P}$-a.s.

$$
\lim _{\varepsilon \rightarrow 0} \mathbf{1}_{A_{\varepsilon}}=\mathbf{1}_{\left\{\sigma_{x}(t)<\infty ; X_{\sigma_{x}(t)}>x\right\}}
$$

and complete the proof by letting $\varepsilon$ go to 0 and using dominated convergence in the left side of (10) and monotone convergence in the right side.

We get a similar result for the reversed path at $\tau_{x}$ on the event $\left\{X_{\tau_{x}}>x\right\}$ :

THEOREM 3.2. - Assume that $\pi$ charges $(0,+\infty)$. Then, for any positive real number $x$ and for any bounded measurable functional $F$ on $\Omega$,

$$
\begin{aligned}
\mathbf{E} & {\left[F\left(\widehat{X}^{\tau_{x}}\right) ; \tau_{x}<\infty ; X_{\tau_{x}}>x\right] } \\
& =\int_{(0,+\infty)} \pi(d r) \int_{0}^{+\infty} d u \mathbf{E}_{r}\left[F\left(X_{\cdot \wedge u}\right) ; x<X_{u} \leqslant x+I_{u}\right] .
\end{aligned}
$$

Remark. - In the subordinator case we get immediately the well-known formula:

$$
\mathbf{E}\left[f\left(X_{\tau_{x}-}, X_{\tau_{x}}\right) ; X_{\tau_{x}}>x\right]=\int_{[0, x]} V(d a) \int_{(x-a,+\infty)} \pi(d r) f(a, a+r),
$$

where $V$ denote the potential measure associated with $X$.

Proof of Theorem 3.2. - Let $f$ be a bounded measurable function on $\mathbb{R}$. Observe that

$$
\begin{aligned}
& \mathbf{E}\left[F\left(\widehat{X}^{\tau_{x}-}\right) f\left(\Delta X_{\tau_{x}}\right) ; \tau_{x}<\infty ; X_{\tau_{x}}>x\right] \\
& =\mathbf{E}\left[\sum_{s \geqslant 0} \mathbf{1}_{\left\{S_{s-} \leqslant x ; \Delta X_{s}+X_{s->x\}}\right.} F\left(\widehat{X}^{s-}\right) f\left(\Delta X_{s}\right)\right] .
\end{aligned}
$$

Apply the compensation formula to get

$$
\begin{aligned}
\mathbf{E} & {\left[F\left(\widehat{X}^{\tau_{x}-}\right) f\left(\Delta X_{\tau_{x}}\right) ; \tau_{x}<\infty ; X_{\tau_{x}}>x\right] } \\
& =\int_{(0,+\infty)} \pi(d r) f(r) \int_{0}^{+\infty} d u \mathbf{E}\left[F\left(\widehat{X}^{u}\right) ; S_{u} \leqslant x ; r+X_{u}>x\right]
\end{aligned}
$$

and the result follows by the duality property. 
Recall that if the Lévy measure charges $(0,+\infty)$, the excursion under the supremum may end with a jump. We now give a decomposition of the law of the excursion under the supremum reversed at its final jump time.

THEOREM 3.3. - Assume (A) and that $\pi$ charges $(0,+\infty)$. Then, for any nonnegative measurable functional $F$ on $\Omega$,

$$
N^{*}\left(F\left(\hat{\omega}^{\zeta}\right) ; \omega_{\zeta}>0\right)=\int_{(0,+\infty)} \pi(d r) \mathbf{E}_{r}\left[\int_{0}^{L_{\infty}} d v F\left(X_{\cdot \wedge L_{v}^{-1}}\right) \mathbf{1}_{\left\{X_{L_{v}^{-1}}>0\right\}}\right] .
$$

Remark. - In the spectrally positive case, if we take $L=-I$, then, $L_{x}^{-1}=\tau_{-x}$. Theorem 3.3 shows that under $N^{*}\left(\cdot \cap\left\{\omega_{\zeta}>0\right\}\right)$, the law of $\omega_{\zeta}$ admits a density with respect to Lebesgue measure that is given by

$$
x \rightarrow \mathbf{1}_{(-\infty, 0)}(x) \mathbf{P}\left(I_{\infty}<x\right) \pi((-x,+\infty)) .
$$

Furthermore, under $N^{*}\left(\cdot \mid \omega_{\zeta-}=-x\right)$, the path $\widehat{\omega}^{\zeta-}$ is distributed as $X X_{\cdot \wedge \tau_{-x}}$ under $\mathbf{P}\left(\cdot \mid \tau_{x}<\infty\right)$. This result has been used by Bertoin in [2] and [1].

Proof of Theorem 3.3. - For any nonnegative measurable function $f$ on $\mathbb{R}$, we have the following decomposition:

$$
N^{*}\left(F\left(\hat{\omega}^{\zeta-}\right) f\left(\Delta \omega_{\zeta}\right) ; \omega_{\zeta}>0\right)=N^{*}\left(\sum_{s \geqslant 0} \mathbf{1}_{\left\{\omega_{s-}+\Delta \omega_{s}>0\right\}} F\left(\hat{\omega}^{s-}\right) f\left(\Delta \omega_{s}\right)\right) .
$$

Observe that $\omega$ under $N^{*}$ is markovian with the transition kernel of the Lévy process killed in $[0,+\infty)$. We can apply the compensation formula to get

$$
N^{*}\left(F\left(\hat{\omega}^{\zeta-}\right) f\left(\Delta \omega_{\zeta}\right) ; \omega_{\zeta}>0\right)=N^{*}\left(\int_{0}^{\zeta} d s F\left(\hat{\omega}^{s}\right) \int_{(0,+\infty)} \pi(d r) f(r) \mathbf{1}_{\left\{\omega_{s}+r>0\right\}}\right)
$$

that yields the theorem thanks to Proposition 2.3.

\section{Applications}

\subsection{First Williams' decomposition theorem}

From now on until the end of the present article, we assume (A) and we suppose that $X$ does not drift to $-\infty$. Let $x$ be a positive real number. Williams has shown in [19] that the standard real Brownian motion reversed at the first hitting time of $(x,+\infty)$ is distributed as the three-dimensional Bessel process up to its last passage time at $x$. In this section, we extend Williams' result to general Lévy processes, the role of the three-dimensional Bessel process being played by the Lévy process conditioned to stay positive. In order to avoid cumbersome notation, we set for any positive real numbers $x$ and $t$ 


$$
\begin{gathered}
\stackrel{\sigma}{\rightarrow}^{t}=\sigma_{x}\left(\stackrel{X}{\rightarrow}^{t}\right)=\sup \left\{s \in\left[0, t-\underline{g}_{t}\right]: X_{s+\underline{g}_{t}}-I_{t} \leqslant x\right\}, \\
\sigma_{x}^{\uparrow}=\sigma_{x}\left(X^{\uparrow}\right)=\sup \left\{s \geqslant 0: X_{s}^{\uparrow} \leqslant x\right\}, \\
\stackrel{\tau}{\tau}^{t}=\tau_{x}\left(\stackrel{X}{\rightarrow}^{t}\right)=\inf \left\{s \in\left[0, t-\underline{g}_{t}\right]: X_{s+\underline{g}_{t}}-I_{t}>x\right\}, \\
\tau_{x}^{\uparrow}=\tau_{x}\left(X^{\uparrow}\right)=\inf \left\{s \geqslant 0: X_{s}^{\uparrow}>x\right\}
\end{gathered}
$$

(with $\inf \emptyset=\sup \emptyset=+\infty$ ). Observe that $\stackrel{\sigma}{\rightarrow}_{x}^{t}$ may be infinite if $X_{t}-\inf _{[0, t]} X<x$. Under $\mathbf{P}\left(\cdot \cap\left\{\stackrel{\sigma}{\rightarrow}^{t}<\infty\right\}\right)$ we define

$$
Y^{t}=\stackrel{X}{\rightarrow} \stackrel{\circ}{\rightarrow} \underset{\rightarrow}{\rightarrow} \stackrel{t}{x}
$$

Similarly we denote

$$
Y=X^{\uparrow} \circ \theta_{\sigma_{x}^{\uparrow}}
$$

that is well-defined thanks to (7) and our assumptions. Notice that $Y^{t}$ and $Y$ rely on $x$ although it does not appear in the notations. We recall that $\underset{\leftarrow}{Y}$ and $\underset{\rightarrow}{\rightarrow}$ are respectively the pre-infimum process and the post-infimum process of $Y$. The following theorems describe the law of the path $X^{\uparrow}$ reversed at time $\sigma_{x}^{\uparrow}$ : the first theorem concerns the case of a jump: $\Delta X_{\sigma_{x}^{\uparrow}}^{\uparrow}>0$; the second theorem deals with the process leaving continuously level $x$.

THEOREM 4.1. - Assume that $\pi$ charges $(0,+\infty)$. Let $x>0$.

(i) $\left({\widehat{X^{\uparrow}}}^{\sigma_{x}^{\uparrow}}, \underset{\leftarrow}{Y}\right)$ under $\mathbf{P}\left(\cdot \mid X_{\sigma_{x}^{\uparrow}}^{\uparrow}>x\right) \stackrel{\text { (law) }}{=}\left(\Delta X_{\tau_{x}}+X_{\cdot \wedge \bar{g}_{\tau_{x}}}, \widehat{X}_{\cdot \wedge\left(\tau_{x}-\bar{g}_{\tau_{x}}\right)}^{\tau_{x}}\right)$ under $\mathbf{P}(\cdot \mid$ $\left.X_{\tau_{x}}>x\right)$.

(ii) Under $\mathbf{P}\left(\cdot \mid X_{\sigma_{x}^{\uparrow}}^{\uparrow}>x\right), \underset{Y}{\rightarrow}$ is independent of $\left(X_{\cdot \wedge \sigma_{x}^{\uparrow}}^{\uparrow} \underset{\leftarrow}{Y}\right)$ and distributed as $X^{\uparrow}$.

THEOREM 4.2. - Assume that $d^{*}>0$. Then, for any $x>0$,

(i) $\mathbf{P}\left(X_{\sigma_{x}^{\uparrow}}^{\uparrow}=x\right)=\mathbf{P}\left(X_{\tau_{x}}=x\right)=d^{*} u^{*}(x)$ and $\widehat{X}^{\tau_{x}}$ under $\mathbf{P}\left(\cdot \mid X_{\tau_{x}}=x\right)$ is distributed as $X_{\cdot \wedge \sigma_{x}^{\uparrow}}^{\uparrow}$ under $\mathbf{P}\left(\cdot \mid X_{\sigma_{x}^{\uparrow}}^{\uparrow}=x\right)$.

(ii) Under $\mathbf{P}\left(\cdot \mid X_{\sigma_{x}^{\uparrow}}^{\uparrow}=x\right), X^{\uparrow} \circ \theta_{\sigma_{x}^{\uparrow}}$ and $X_{\cdot \wedge \sigma_{x}^{\uparrow}}^{\uparrow}$ are mutually independent and $X^{\uparrow} \circ \theta_{\sigma_{x}^{\uparrow}}$ is distributed as $X^{\uparrow}$.

Remark. - We assume that $\pi$ charges $(-\infty, 0)$. Then, the excursion under the infimum may end with a negative jump. The dual form of the reversion formula of Theorem 3.3 gives

$$
N\left(F\left(\omega_{\cdot \wedge \zeta-}\right) ; \omega_{\zeta}<0\right)=\int_{(-\infty, 0)} \pi(d r) \mathbf{E}\left[\int_{0}^{L_{\infty}^{*}} d u \mathbf{1}_{\left\{X_{L_{u}^{*-1}}<-r\right\}} F\left(\widehat{X}^{L_{u}^{*-1}}\right)\right]
$$


We assume moreover that $d^{*}>0$. By Lemma 2.1, it follows that under $N\left(\cdot \cap\left\{\omega_{\zeta}<0\right\}\right)$, $\omega_{\zeta-}$ admits a density with respect to Lebesgue measure given by

$$
N\left(\omega_{\zeta-} \in d x ; \omega_{\zeta}<0\right)=u^{*}(x) \pi((-\infty,-x)) \mathrm{m}(d x) .
$$

By combining (11) with Lemma 2.1, Theorem 4.2 implies that for any $x>0$,

$$
X_{\cdot \wedge \sigma_{x}^{\uparrow}}^{\uparrow} \text { under } \mathbf{P}\left(\cdot \mid X_{\sigma_{x}^{\uparrow}}^{\uparrow}=x\right) \stackrel{(\text { law })}{=} \omega_{\cdot \wedge \zeta-} \text { under } N\left(\cdot \mid \omega_{\zeta-}=x\right) .
$$

This result is due to Chaumont in the stable case (see [7]).

Proof of Theorem 4.1. - First observe that $\tau_{x}(J)=\sigma_{x}^{\uparrow}$ a.s., then by Lemma 2.5

$$
\sigma_{x}^{\uparrow} \stackrel{\text { (law) }}{=} \inf \left\{t>0: S_{\bar{d}_{t}}>x\right\}=\bar{g}_{\tau_{x}} \text {. }
$$

Set

$$
\gamma=\sigma_{x}^{\uparrow}+\underline{g}(Y)=\inf \left\{t>\sigma_{x}^{\uparrow}: X_{t}^{\uparrow}=J_{t}\right\} .
$$

Then,

$$
\gamma \stackrel{(\text { law })}{=} \tau_{x}
$$

We define the functional $\Sigma$ by

$$
\Sigma_{t}(X)=\left(S_{\left(\bar{d}_{t}+\bar{g}_{t}-t\right)-}-X_{\left(\bar{d}_{t}+\bar{g}_{t}-t\right)-}, S_{\bar{d}_{t}}\right), \quad t \geqslant 0 .
$$

Deduce from (12) and from the fact that $\bar{d}_{\tau_{x}}=\tau_{x}$ that

$$
\left(\left(X^{\uparrow}-J\right)_{\gamma+t}, J_{\gamma+t}-J_{\gamma}\right)_{t \geqslant 0} \stackrel{\text { (law) }}{=}\left(\Sigma_{t}\left(X \circ \theta_{\tau_{x}}\right)\right)_{t \geqslant 0} .
$$

Since

$$
\stackrel{Y}{\rightarrow}_{t}=\left(X^{\uparrow}-J\right)_{\gamma+t}+\left(J_{\gamma+t}-J_{\gamma}\right)
$$

we deduce from the Markov property applied at $\tau_{x}$ in the right member of (13) that $\stackrel{Y}{\rightarrow}$ is independent of $X_{\bullet \wedge \gamma}^{\uparrow}$ and that $\underset{\rightarrow}{\rightarrow}$ has the same distribution as $X^{\uparrow}$, which proves Theorem 4.1(ii) and also Theorem 4.2(ii) because

$$
X^{\uparrow} \circ \theta_{\sigma_{x}^{\uparrow}}=\underset{Y}{\rightarrow} \text { on }\left\{X_{\sigma_{x}^{\uparrow}}^{\uparrow}=x\right\} .
$$

Next, we denote by $K^{-1}$ the right-continuous inverse of $K$ (recall notation from Section 2.2):

$$
K_{u}^{-1}=\inf \left\{t \geqslant 0: K_{t}>u\right\}, \quad u \geqslant 0 .
$$

We need the following lemma: 
LEMMA 4.3. - For any $x>0$

$$
X_{\cdot \wedge K_{x}^{-1}}^{\uparrow} \stackrel{(\text { law) }}{=} \widehat{X}^{L_{x}^{*-1}}
$$

Proof. - We first index the excursions of $X^{\uparrow}-J$ above 0 by the corresponding local time: for any $t \geqslant 0$, we set

$$
e_{t}^{\uparrow}=\left(\left(X^{\uparrow}-J\right)_{\left(K_{t-}^{-1}+s\right) \wedge K_{t}^{-1}}\right)_{s \geqslant 0}
$$

(note that $e_{t}^{\uparrow}=0$ if $\Delta K_{t}^{-1}=0$ ). We do the same thing for the excursions of $X$ under its supremum and we set

$$
e_{t}=\left((X-S)_{\left.\left(L_{t-}^{*-1}+s\right) \wedge L_{t}^{*-1}\right)_{s \geqslant 0}} .\right.
$$

We also write $U_{t}^{\uparrow}=X_{K_{t}^{-1}}^{\uparrow}$. We deduce from Lemma 2.5 that $\left(K^{-1}, U^{\uparrow}\right)$ is a subordinator with the same distribution as $\left(L^{*-1}, U^{*}\right)$. There exists a measurable functional $F$ such that

$$
F\left(\left(e_{t}^{\uparrow}, U_{t}^{\uparrow}\right)_{0 \leqslant t \leqslant x}\right)=X_{\cdot \wedge K_{x}^{-1}}^{\uparrow}, \quad \text { a.s. }
$$

Let us explain more precisely how to recover $X^{\uparrow}$ from the $e_{t}^{\uparrow}$ and $U_{t}^{\uparrow}, t \geqslant 0$ : For any $s \in\left[0, K_{x}^{-1}\right]$, we define

$$
g(s)=\sup \left\{u \in[0, s): X_{u}^{\uparrow}=J_{u}\right\} .
$$

The Lévy-Ito decomposition for the subordinator $K^{-1}$ implies that

$$
K_{s}=\sup \left\{a \in[0, x]: d^{*} a+\sum_{t<a} \zeta\left(e_{t}^{\uparrow}\right) \leqslant s\right\}
$$

and

$$
g(s)=d^{*} K_{s}+\sum_{t<K_{s}} \zeta\left(e_{t}^{\uparrow}\right)
$$

Then,

$$
X_{s}^{\uparrow}=e_{K_{s}}^{\uparrow}(s-g(s))+U_{K_{s}}^{\uparrow} .
$$

In order to simplify notations, we set for any $t \geqslant 0$ :

$$
\hat{e}_{t}=\hat{e}_{t}^{\zeta\left(e_{t}\right)-} \text {. }
$$

Lemma 2.5 implies

$$
\left(e_{t}^{\uparrow}, U_{t}^{\uparrow}\right)_{0 \leqslant t \leqslant x} \stackrel{\text { (law) }}{=}\left(\hat{e}_{t}, U_{t}^{*}\right)_{0 \leqslant t \leqslant x} .
$$

Since $\left(e_{t} ; t \geqslant 0\right)$ is a Poisson process and $U^{*}$ a subordinator, a simple time-reversal argument show that

$$
\left(e_{x-t}, \widehat{U}^{*}{ }_{t}^{x}\right)_{0 \leqslant t \leqslant x} \stackrel{\text { (law) }}{=}\left(e_{t}, U_{t}^{*}\right)_{0 \leqslant t \leqslant x}
$$


Thus

$$
\left(e_{t}^{\uparrow}, U_{t}^{\uparrow}\right)_{0 \leqslant t \leqslant x} \stackrel{(\text { law })}{=}\left(\hat{e}_{x-t}, \widehat{U}^{*}{ }_{t}^{x}\right)_{0 \leqslant t \leqslant x} .
$$

Applying the Lévy-Ito decomposition for the subordinator $L^{*-1}$ reversed at time $x$, it is easy to check that

$$
F\left(\left(\hat{e}_{x-t}, \widehat{U}^{*}{ }_{t}^{x}\right)_{0 \leqslant t \leqslant x}\right)=\widehat{X}^{L_{x}^{*-1}}, \quad \text { a.s. }
$$

and we conclude thanks to (15).

Let us prove now Theorem 4.1(i): Let $F$ and $G$ be two nonnegative measurable functionals and $f$ be a nonnegative measurable function. Set

$$
\alpha=\mathbf{E}\left[F\left(X_{\cdot \wedge \sigma_{x}^{\uparrow-}}^{\uparrow}\right) f\left(\Delta X_{\sigma_{x}^{\uparrow}}^{\uparrow}\right) G(\underset{\leftarrow}{Y}) ; X_{\sigma_{x}^{\uparrow}}^{\uparrow}>x\right] .
$$

It is sufficient to show that

$$
\alpha=\mathbf{E}\left[F\left(\widehat{X}^{\bar{g}_{\tau_{x}}}\right) f\left(\Delta X_{\tau_{x}}\right) G\left(\widehat{X}_{\cdot \wedge\left(\tau_{x}-\bar{g}_{\tau_{x}}\right.}^{\tau_{\tau_{x}}}\right) ; X_{\tau_{x}}>x\right] .
$$

First observe that

$$
\alpha=\mathbf{E}\left[\sum_{i \in \mathcal{I} \uparrow} \mathbf{1}_{\left\{X_{g_{i}}^{\uparrow} \leqslant x<X_{g_{i}}^{\uparrow}+w^{i}(0)\right\}} F\left(X_{\cdot \wedge g_{i}}^{\uparrow}\right) f\left(w^{i}(0)\right) G\left(w^{i}-w^{i}(0)\right)\right] .
$$

Then by (8), we get

$$
\alpha=\int_{0}^{\infty} d u \mathbf{E}\left[F\left(X_{\cdot \wedge K_{u}^{-1}}^{\uparrow}\right) \widehat{N}^{*}\left(f(w(0)) G(w-w(0)) ; U_{u}^{\uparrow} \leqslant x<U_{u}^{\uparrow}+w(0)\right)\right] .
$$

The previous lemma implies that

$$
\alpha=\int_{0}^{\infty} d u \mathbf{E}\left[F\left(\widehat{X}^{L_{u}^{*-1}}\right) N^{*}\left(f\left(\Delta \omega_{\zeta}\right) G\left(\widehat{\omega}^{\zeta-}\right) ; U_{u}^{*} \leqslant x<U_{u}^{*}+\omega(\zeta)\right)\right] .
$$

But we have a.s.

$$
\begin{aligned}
& \mathbf{1}_{\left\{X_{\left.\tau_{x}>x\right\}}\right.} F\left(\widehat{X}^{\bar{g}_{\tau_{x}}}\right) f\left(\Delta X_{\tau_{x}}\right) G\left(\widehat{X}_{\cdot \wedge\left(\tau_{x}-\bar{g}_{\tau_{x}}\right)}^{\tau_{x}}\right) \\
& =\sum_{i \in \mathcal{I}^{*}} \mathbf{1}_{\left\{X_{g_{i}} \leqslant x<X_{g_{i}}+\omega^{i}\left(\zeta_{i}\right)\right\}} F\left(\widehat{X}^{g_{i}}\right) f\left(\Delta \omega_{\zeta_{i}}^{i}\right) G\left({\widehat{\omega^{i}}}^{\zeta_{i}-}\right) .
\end{aligned}
$$

Then, the compensation formula combined with (17) achieve the proof of (16).

Proof of Theorem 4.2. - We only need to show (i). From Theorem 4.1, we deduce that

$$
\mathbf{P}\left(X_{\sigma_{x}^{\uparrow}}^{\uparrow}>x\right)=\mathbf{P}\left(X_{\tau_{x}}>x\right) .
$$


So, we have for any $x>0$ :

$$
d^{*} u^{*}(x)=\mathbb{P}\left(X_{\sigma_{x}^{\uparrow}}^{\uparrow}=x\right) .
$$

We need the following lemma:

LEMMA 4.4. - Under the assumptions of Theorem 4.2, we have for any nonnegative measurable functional $F$

$$
\mathbf{E}\left[\int_{0}^{\infty} d u F\left(X_{\cdot \wedge K_{u}^{-1}}^{\uparrow}\right)\right]=\int_{0}^{\infty} d x u^{*}(x) \mathbf{E}\left[F\left(X_{\cdot \wedge \sigma_{x}^{\uparrow}}^{\uparrow}\right) \mid X_{\sigma_{x}^{\uparrow}}^{\uparrow}=x\right] .
$$

Proof. - We argue exactly as in Lemma 2.1 replacing, $X$ by $X^{\uparrow}, L^{*}$ by $K$ and $\tau_{x}$ by $\sigma_{x}^{\uparrow}$.

Lemmas 2.1 and 4.4 imply that for any nonnegative measurable functional $F$, the set

$$
\lambda_{F}=\left\{x>0: \mathbf{E}\left[F\left(X_{\cdot \wedge \sigma_{x}^{\uparrow}}^{\uparrow}\right) ; X_{\sigma_{x}^{\uparrow}}^{\uparrow}=x\right]=\mathbf{E}\left[F\left(\widehat{X}^{\tau_{x}}\right) ; X_{\tau_{x}}=x\right]\right\}
$$

is of full Lebesgue measure. We have to show that actually $\lambda_{F}=(0,+\infty)$ : Let $x_{0}>0$, let $G$ be such that for any $x>0$ :

$$
G\left(X_{\cdot \wedge \sigma_{x}^{\uparrow}}^{\uparrow}\right)=\mathbf{1}_{\left(x_{0}, \infty\right)}(x) F\left(X_{\cdot \wedge \sigma_{x_{0}}^{\uparrow}}^{\uparrow}\right) \mathbf{1}_{\left\{X_{\sigma_{x_{0}}^{\uparrow}}^{\uparrow}=x_{0}\right\}} .
$$

Observe that on $\left\{X_{\sigma_{x_{0}}^{\uparrow}}^{\uparrow}=x_{0}\right\}$, we have for any $x>x_{0}$

$$
\sigma_{x}^{\uparrow}=\sigma_{x-x_{0}}\left(X^{\uparrow} \circ \theta_{\sigma_{x_{0}}^{\uparrow}}\right)+\sigma_{x_{0}}^{\uparrow} .
$$

Then, Theorem 4.1(ii) (already proved) implies that

$$
\mathbf{E}\left[G\left(X_{\cdot \wedge \sigma_{x}^{\uparrow}}^{\uparrow}\right) ; X_{\sigma_{x}^{\uparrow}}^{\uparrow}=x\right]=d^{*} u^{*}\left(x-x_{0}\right) \mathbf{E}\left[F\left(X_{\cdot \wedge \sigma_{x_{0}}^{\uparrow}}^{\uparrow}\right) ; X_{\sigma_{x_{0}}^{\uparrow}}^{\uparrow}=x_{0}\right] .
$$

Since $\lambda_{G}$ is a set of full Lebesgue measure we can assume that $x>x_{0}$ is in $\lambda_{G}$ and consequently

$$
\mathbf{E}\left[G\left(X_{\cdot \wedge \sigma_{x}^{\uparrow}}^{\uparrow}\right) ; X_{\sigma_{x}^{\uparrow}}^{\uparrow}=x\right]=\mathbf{E}\left[G\left(\widehat{X}^{\tau_{x}}\right) ; X_{\tau_{x}}=x\right] .
$$

But

$$
\mathbf{1}_{\left\{X_{\tau_{x}}=x\right\}} G\left(\widehat{X}^{\tau_{x}}\right)=F\left(\widehat{Z}^{\tau_{x_{0}}(Z)}\right) \mathbf{1}_{\left\{Z_{\tau_{x_{0}}(Z)=x_{0}} \text { and } X_{\tau_{x-x_{0}}}=x-x_{0}\right\}},
$$

where $Z=X \circ \theta_{\tau_{x-x_{0}}}$. Applying the Markov property at time $\tau_{x-x_{0}}$, we get that

$$
\mathbf{E}\left[G\left(X_{\cdot \wedge \sigma_{x}^{\uparrow}}^{\uparrow}\right) ; X_{\sigma_{x}^{\uparrow}}^{\uparrow}=x\right]=d^{*} u^{*}\left(x-x_{0}\right) \mathbf{E}\left[F\left(\widehat{X}^{\tau_{x_{0}}}\right) ; X_{\tau_{x_{0}}}=x_{0}\right]
$$

which implies the desired result. 


\subsection{Bismut's decomposition}

As a consequence of Theorem 4.2 and Lemma 2.1, we extend to real Lévy processes Bismut's decomposition of the excursion above the infimum.

THEOREM 4.5. - Assume that $d^{*}$ is positive. Then for any nonnegative measurable functionals $G$ and $D$ on $\Omega$ and any nonnegative measurable function $f$,

$$
\begin{aligned}
& N\left(\int_{0}^{\zeta} d s G\left(\omega_{\cdot \wedge s}\right) f\left(\omega_{s}\right) D\left(\omega \circ \theta_{s}\right)\right) \\
& \quad=\int_{0}^{+\infty} d x f(x) u^{*}(x) \mathbf{E}\left[G\left(X_{\cdot \wedge \sigma_{x}^{\uparrow}}^{\uparrow}\right) \mid X_{\sigma_{x}^{\uparrow}}^{\uparrow}=x\right] \mathbf{E}\left[D\left(X_{\cdot \wedge \tau_{-x}}\right)\right] .
\end{aligned}
$$

Remark. - The spectrally positive case is due to Chaumont (see [9]).

Proof. - Apply Markov property under $N$ in order to get:

$$
N\left(\int_{0}^{\zeta} d s G\left(\omega_{\cdot \wedge s}\right) f\left(\omega_{s}\right) D\left(\omega \circ \theta_{s}\right)\right)=N\left(\int_{0}^{\zeta} d s G\left(\omega_{\cdot \wedge s}\right) f\left(\omega_{s}\right) d\left(\omega_{s}\right)\right),
$$

where, for any positive number $x, d(x)$ stands for $\mathbf{E}\left[D\left(X_{\cdot \wedge \tau_{-x}}\right)\right]$. Then, by Proposition 2.3, we have

$$
\begin{aligned}
& N\left(\int_{0}^{\zeta} d s G(\omega \cdot \wedge s) f\left(\omega_{s}\right) D\left(\omega \circ \theta_{s}\right)\right) \\
& =\mathbf{E}\left[\int_{0}^{L_{\infty}^{*}} d u G\left(\widehat{X}^{L_{u}^{*-1}}\right) f\left(X_{L_{u}^{*-1}}\right) d\left(X_{L_{u}^{*-1}}\right)\right]
\end{aligned}
$$

and we use Lemma 2.1 and Theorem 4.2 to complete the proof.

We have seen in Section 3 that the excursion under the supremum may end with a jump if $\pi$ charges $(0,+\infty)$. Theorem 3.3 provides a reversion formula for the excursion under the supremum at its final jump time. If we assume that $d^{*}$ is positive, then, the excursion may end continuously, as to say $\omega_{\zeta}=0$. More precisely it is clear that $N^{*}\left(\omega_{\zeta}=0\right)=0$ if $d^{*}=0$; let us show that $N^{*}\left(\omega_{\zeta}=0\right)=+\infty$ if $d^{*}>0$ :

$$
\begin{aligned}
N^{*}\left(1-e^{-\lambda \zeta} ; \omega_{\zeta}=0\right) & =\lambda \int_{0}^{+\infty} d s e^{-\lambda s} N^{*}\left(\zeta>s ; \omega_{\zeta}=0\right) \\
& =\lambda \int_{0}^{+\infty} d s e^{-\lambda s} N^{*}\left(\left[\mathbf{P}\left(X_{\tau_{a}}=a\right)\right]_{a=-\omega_{s}} ; \zeta>s\right) \\
& =\lambda \int_{0}^{+\infty} d s e^{-\lambda s} N^{*}\left(d^{*} u^{*}\left(-\omega_{s}\right) ; \zeta>s\right) .
\end{aligned}
$$


By a change of variable, we have

$$
\lambda \int_{0}^{+\infty} d s e^{-\lambda s} N^{*}\left(d^{*} u^{*}\left(-\omega_{s}\right) ; \zeta>s\right)=\int_{0}^{+\infty} d s e^{-s} N^{*}\left(d^{*} u^{*}\left(-\omega_{s / \lambda}\right) ; \zeta>s / \lambda\right) .
$$

Then for any $\lambda>0$, we have

$$
N^{*}\left(1-e^{-\lambda \zeta} ; \omega_{\zeta}=0\right) \geqslant \frac{d^{*}}{e} \inf _{x \in(0,1]} u^{*}(x) N^{*}\left(\sup _{s \in[0,1 / \lambda]}\left(-\omega_{s}\right) \leqslant 1 ; \zeta>1 / \lambda\right) .
$$

But

$$
\begin{aligned}
& \lim _{\lambda \rightarrow+\infty} N^{*}\left(1-e^{-\lambda \zeta} ; \omega_{\zeta}=0\right) \\
& \quad=N^{*}\left(\omega_{\zeta}=0\right) \geqslant \frac{d^{*}}{e} \inf _{x \in(0,1]} u^{*}(x) \lim _{\lambda \rightarrow+\infty} N^{*}\left(\sup _{s \in[0,1 / \lambda]}\left(-\omega_{s}\right) \leqslant 1 ; \zeta>1 / \lambda\right)=+\infty .
\end{aligned}
$$

The following theorem complements Theorem 3.3 by providing a reversion identity for the excursion under the supremum ending continuously.

THEOREM 4.6. - Assume that $d d^{*}>0$. Then, for any nonnegative measurable functional $F$ on $\Omega$,

$$
d N^{*}\left(F\left(\hat{\omega}^{\zeta}\right) ; \omega_{\zeta}=0\right)=d^{*} N\left(F(\omega) ; \omega_{\zeta}=0\right) .
$$

Remark. - The theorem remains true if $d^{*} d=0$ : in that case, it just means that either $N^{*}\left(\omega_{\zeta}=0\right)=0$ or $N\left(\omega_{\zeta}=0\right)=0$.

Proof. - We prove the following identity:

$$
d N^{*}\left(\int_{0}^{\zeta} d s F\left(\hat{\omega}_{\cdot \wedge s}^{\zeta}\right) ; \omega_{\zeta}=0\right)=d^{*} N\left(\int_{0}^{\zeta} d s F\left(\omega_{\cdot \wedge s}\right) ; \omega_{\zeta}=0\right),
$$

which easily leads to the statement of the theorem. First, observe that

$$
\widehat{\omega}_{\cdot \wedge s}^{\zeta}=\widehat{\omega \circ \theta_{\zeta-s}} .
$$

After the change of variable $s \rightarrow \zeta-s$, the Markov property under $N^{*}$ combined with the latter observation give

$$
d N^{*}\left(\int_{0}^{\zeta} d s F\left(\hat{\omega}_{\cdot \wedge s}^{\zeta}\right) ; \omega_{\zeta}=0\right)=d N^{*}\left(\int_{0}^{\zeta} d s \mathbf{E}\left[F\left(\widehat{X}^{\tau_{-\omega_{s}}}\right) ; X_{\tau_{-\omega_{s}}}=-\omega_{s}\right]\right) .
$$

By Proposition 2.3 and the dual version of Lemma 2.1, it follows that

$$
d N^{*}\left(\int_{0}^{\zeta} d s F\left(\hat{\omega}_{\cdot \wedge s}^{\zeta}\right) ; \omega_{\zeta}=0\right)=d \int_{0}^{+\infty} d x u(-x) \mathbf{E}\left[F\left(\widehat{X}^{\tau_{x}}\right) ; X_{\tau_{x}}=x\right]
$$




$$
=d d^{*} \int_{0}^{+\infty} d x u^{*}(x) u(-x) \mathbf{E}\left[F\left(\widehat{X}^{\tau_{x}}\right) \mid X_{\tau_{x}}=x\right] .
$$

Use Proposition 2.3 and Lemma 2.1 to get

$$
d d^{*} \int_{0}^{+\infty} d x u^{*}(x) u(-x) \mathbf{E}\left[F\left(\widehat{X}^{\tau_{x}}\right) \mid X_{\tau_{x}}=x\right]=d^{*} N\left(\int_{0}^{\zeta} d s F\left(\omega_{\cdot \wedge s}\right) ; \omega_{\zeta}=0\right)
$$

that is the desired result.

\subsection{The second Williams' decomposition theorem}

Williams has shown in [19] that the Brownian excursion splits at its maximum in two three-dimensional Bessel processes stopped at a certain hitting time. In this section, we extend this result to general Lévy processes. To simplify, we set

$$
Z^{T}=\underset{X^{X}}{\rightarrow} \stackrel{\theta_{\underset{\tau}{x}}^{T}}{\rightarrow} \text { under } \mathbf{P}\left(\cdot \cap\left\{\stackrel{\sim \tau}{\sim}^{T}<\infty\right\}\right) \text { and } Z=X^{\uparrow} \circ \theta_{\tau_{x}^{\uparrow}} .
$$

We first prove the following proposition.

Proposition 4.7. - (i) For any bounded measurable functional $F$ on $\Omega$,

$$
\mathbf{E}\left[F\left(X_{\cdot \wedge \tau_{x}^{\uparrow}}^{\uparrow}\right)\right]=N\left(F\left(\omega_{\cdot \wedge \tau_{x}(\omega)}\right) \mathcal{U}\left(\left(-\omega_{\tau_{x}(\omega)}, 0\right]\right) ; \tau_{x}(\omega)<\infty\right) .
$$

Thus, $\mathbf{P}\left(X_{\tau_{x}^{\uparrow}}^{\uparrow}=x\right)>0$ if and only if $d^{*}>0$.

(ii) If $d^{*}>0$, then, under $\mathbf{P}\left(\cdot \mid X_{\tau_{x}^{\uparrow}}^{\uparrow}=x\right), \underset{\leftarrow}{Z}$ and $\underset{Z}{Z}$ are mutually independent; the process $\underset{Z}{\rightarrow}$ has the same law as $X^{\uparrow}$ and the law of $\underset{\leftarrow}{Z}$ is characterized by the following identity that holds for any nonnegative measurable functional $F$ on $\Omega$ :

$$
\mathbf{E}[F(\underset{\leftarrow}{Z})]=\frac{1}{\mathcal{U}((-x, 0])} \mathbf{E}\left[\int_{0}^{L_{\infty}} d v F\left(X_{\cdot \wedge L_{v}^{-1}}\right) \mathbf{1}_{\left\{X_{L_{v}^{-1}}>-x\right\}}\right] .
$$

Remark. - Recall from Section 2.1, that under $\mathbf{P}\left(\cdot \mid X_{\tau_{x}^{\uparrow}}^{\uparrow}=x\right)$, the process $x+Z$ is markovian with a transition kernel given by

$$
p_{t}^{+}(y, d z)=\frac{\mathcal{U}((-z, 0])}{\mathcal{U}((-y, 0])} q_{t}^{+}(y, d z), \quad y \geqslant 0,
$$

where $q_{t}^{+}$stands for the semigroup of the Lévy process killed in $(-\infty, 0]$. Then, the latter proposition combined with Lemma 2.1 gives the following corollary.

COROLlary 4.8. - Assume that $d d^{*}>0$. Let $X^{\uparrow}(x)$ denote the Lévy process started at $x>0$ and conditioned to stay positive. The path $X^{\uparrow}(x)$ has the following decomposition at its infimum: 
(i) The pre-infimum process $\underset{\leftarrow}{X^{\uparrow}}(x)$ and the post-infimum process $\underset{\rightarrow}{\longrightarrow}(x)$ are mutually independent and ${\underset{X}{\uparrow}}^{\uparrow}(x)$ is distributed as the Lévy process conditioned to stay positive started at 0 .

(ii) The law of the infimum of $X^{\uparrow}(x)$ admits a density with respect to Lebesgue measure that is given by:

$$
y \rightarrow \mathbf{1}_{[0, x]}(y) \frac{u(y-x)}{\mathcal{U}((-x, 0])}
$$

and under $\mathbf{P}\left(\cdot \mid \inf X^{\uparrow}(x)=y\right),{\underset{\leftarrow}{\uparrow}}^{\Upsilon}(x)$ is distributed as $x+X_{\cdot \wedge \tau_{y-x}}$ under $\mathbf{P}\left(\cdot \mid X_{\tau_{y-x}}=\right.$ $y-x)$.

Remarks. - (i) We can actually show that the corollary remains true even if $d^{*}=0$.

(ii) When $X$ does not drift to $-\infty$ the result is Theorem 5 of Chaumont [9] (see also [7] and [8]).

Proof of Proposition 4.7. - Let $T$ be independent of $X$ and exponentially distributed with parameter $\alpha$. Recall from Lemma 2.2 that for any nonnegative measurable functional $H$ defined on $\Omega$,

$$
\mathbf{E}\left[H\left(\underline{X}^{T}\right)\right]=\frac{\alpha}{\kappa(\alpha, 0)} N\left(\int_{0}^{\zeta} d s e^{-\alpha s} H(\omega \cdot \wedge s)\right) .
$$

Let $G$ and $D$ be nonnegative measurable functionals on $\Omega$. Take

$$
H(\omega)=\mathbf{1}_{\left\{\tau_{x}(\omega)<\infty\right\}} G\left(\omega\left(\cdot \wedge \tau_{x}(\omega)\right)\right) D\left(\omega \circ \theta_{\left.\tau_{x}(\omega)\right)}\right), \quad \omega \in \Omega .
$$

Then,

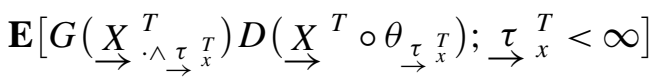

$$
\begin{aligned}
& =\frac{\alpha}{\kappa(\alpha, 0)} N\left(\mathbf{1}_{\left\{\tau_{x}(\omega)<\infty\right\}} G\left(\omega \cdot \wedge \tau_{x}(\omega)\right) e^{-\alpha \tau_{x}(\omega)} \int_{0}^{\zeta-\tau_{x}(\omega)} d s e^{-\alpha s} D\left(\left(\omega \circ \theta_{\tau_{x}(\omega)}\right) \cdot \wedge s\right)\right) .
\end{aligned}
$$

Apply the Markov property under $N$ in order to get

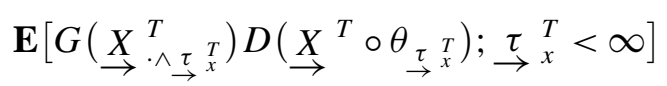

$$
\begin{aligned}
& =N\left(\mathbf{1}_{\left\{\tau_{x}(\omega)<\infty\right\}} e^{-\alpha \tau_{x}(\omega)} G\left(\omega_{\cdot \wedge \tau_{x}(\omega)}\right) d_{\alpha}\left(\omega_{\tau_{x}(\omega)}\right)\right),
\end{aligned}
$$

where for any $a>0$,

$$
d_{\alpha}(a)=\frac{1}{\kappa(\alpha, 0)} \mathbf{E}\left[D\left(X_{\cdot \wedge T}\right) ; I_{T}>-a\right] .
$$


Take $D=1$ in (20). We get

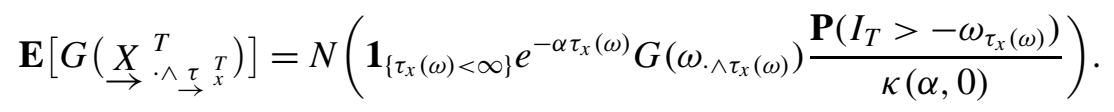

Next we need the following lemma:

Lemma 4.9. - Let $\alpha>0$ and $T_{\alpha}$ be independent of $X$ and exponentially distributed with parameter $\alpha$. Let $F, G$ and $K$ be three bounded measurable functionals on $\Omega$. Under the same assumptions as Theorem 4.1, we have

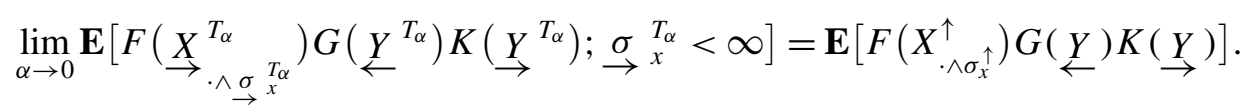

Proof. - It is sufficient to show the limit



We use the notation of Section 2.2. Recall that $A_{s}^{+}=\int_{0}^{s} d u \mathbf{1}_{\left\{X_{u}>0\right\}}$. From Theorem 2.4, we have

$$
\left(\stackrel{X}{\rightarrow}^{s}\right)_{0 \leqslant u<s-\underline{g}} \stackrel{(\text { law) }}{=}\left(X_{u}^{\uparrow}\right)_{0 \leqslant u<A_{s}^{+}} .
$$

Set $\beta=\sigma_{x}^{\uparrow}+g(Y)$. By (7), $\beta<\infty$. In order to avoid cumbersome notations, we denote by $W$ and $W^{\prime}$ respectively the pre-infimum process and the post-infimum process of $X^{\uparrow} \circ \theta_{\sigma_{x}\left(X^{\uparrow}, A_{s}^{+}\right)}$. Let $M>0$ be an upper bound for $F, G$ and $K$. Observe that on $\left\{A_{s}^{+}>\beta\right\}$, we have $\sigma_{x}\left(X^{\uparrow}, A_{s}^{+}\right)=\sigma_{x}^{\uparrow}$ and

$$
F\left(X_{\cdot \wedge \sigma_{x}\left(X^{\uparrow}, A_{s}^{+}\right)}^{\uparrow}\right) G(W) K\left(W^{\prime}\right)=F\left(X_{\cdot \wedge \sigma_{x}^{\uparrow}}^{\uparrow}\right) G(\underset{\leftarrow}{Y}) K(\underset{\rightarrow}{\rightarrow}) .
$$

Then by (21) and (22), we have

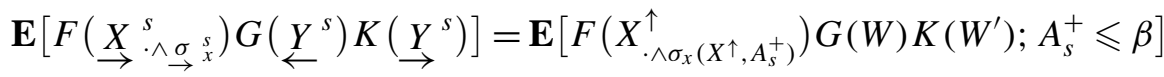

$$
\begin{aligned}
& +\mathbf{E}\left[F\left(X_{\cdot \wedge \sigma_{x}^{\uparrow}}^{\uparrow}\right) G(\underset{\leftarrow}{Y}) K(\underset{Y}{\rightarrow}) ; A_{s}^{+}>\beta\right] .
\end{aligned}
$$

Consequently,

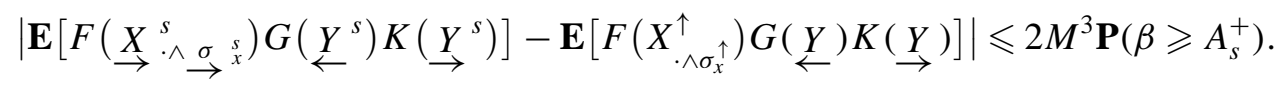

But $\lim _{s \rightarrow \infty} A_{s}^{+}=\infty$. So $\lim _{s \rightarrow \infty} \mathbf{P}\left(\beta \geqslant A_{s}^{+}\right)=0$ which yields the lemma.

Let us achieve the proof of the proposition: Lemma 4.9 implies

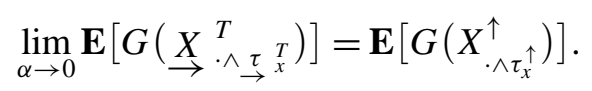


Next, we deduce from Lemma 2.2 that for any $a>0$

$$
\lim _{\alpha \rightarrow 0} \frac{\mathbf{P}\left(I_{T}>-a\right)}{\kappa(\alpha, 0)}=\mathbf{E}\left[\int_{0}^{L^{\infty}} d v \mathbf{1}_{\left\{X_{L_{v}^{-1}}>-a\right\}}\right]=\mathcal{U}((-a, 0]),
$$

which yields (i) by dominated convergence.

Assume now that the drift coefficient $d^{*}$ is positive. Let $D_{1}$ and $D_{2}$ be two nonnegative measurable functionals on $\Omega$. Take,

$$
D(\omega)=D_{1}(\stackrel{\omega}{\leftarrow}) D_{2}(\stackrel{\omega}{\rightarrow}) \text { and } \quad G(\omega)=\mathbf{1}_{\left\{\tau_{x}(\omega)<\infty ; \omega\left(\tau_{x}(\omega)\right)=x\right\}},
$$

in (20). From Lemma 2.2, we note that

$$
\begin{aligned}
d_{\alpha}(a) & =\frac{1}{\kappa(\alpha, 0)} \mathbf{E}\left[D_{1}\left({\underset{X}{\leftarrow}}^{T}\right) ; I_{T}>-a\right] \mathbf{E}\left[D_{2}\left(\underline{X}^{T}\right)\right] \\
& =\mathbf{E}\left[\int_{0}^{L_{\infty}} d v e^{-\alpha L_{v}^{-1}} D_{1}\left(X_{\cdot \wedge L_{v}^{-1}}\right) \mathbf{1}_{\left\{X_{L_{v}^{-1}}>-a\right\}}\right] \mathbf{E}\left[D_{2}\left(\stackrel{X}{\rightarrow}^{T}\right)\right] .
\end{aligned}
$$

Thus, (20) gives

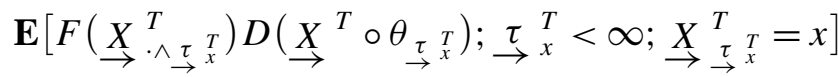

$$
\begin{aligned}
& =\mathbf{E}\left[D_{2}\left(\stackrel{X}{\rightarrow}^{T}\right)\right] N\left(e^{-\alpha \tau_{x}(\omega)} ; \tau_{x}(\omega)<\infty ; \omega_{\tau_{x}(\omega)}=x\right) \\
& \times \mathbf{E}\left[\int_{0}^{L_{\infty}} d v e^{-\alpha L_{v}^{-1}} D_{1}\left(X_{\cdot \wedge L_{v}^{-1}}\right) \mathbf{1}_{\left\{X_{L_{v}^{-1}}>-x\right\}}\right] .
\end{aligned}
$$

To get (ii), pass to the limit $\alpha \rightarrow 0$ in (23) using Lemma 4.9 to write:

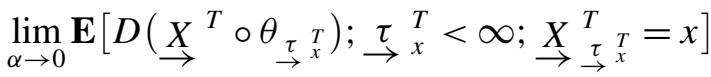

$$
\begin{aligned}
& =\mathbf{E}\left[F\left(X_{\cdot \wedge \tau_{x}^{\uparrow}}^{\uparrow}\right) D_{1}(\underset{\leftarrow}{Z}) D_{2}(\underset{Z}{Z}) ; X_{\tau_{x}^{\uparrow}}^{\uparrow}=x\right] .
\end{aligned}
$$

and

$$
\lim _{\alpha \rightarrow 0} \mathbf{E}\left[D_{2}\left(\stackrel{X}{\rightarrow}^{T}\right)\right]=\mathbf{E}\left[D_{2}\left(X^{\uparrow}\right)\right]
$$

We are now able to state the second Williams' decomposition theorem.

THEOREM 4.10. - Assume that $\pi$ charges $(0,+\infty)$. Suppose also that $d^{*}>0$ and that $X$ oscillates.

(i) The law of $\omega_{\bar{g}(\omega)}$ under $N$ admits a density with respect to Lebesgue measure that is given by

$$
x \rightarrow \frac{1}{d^{*}} N^{*}\left(\tau_{-x}(\omega)<\infty\right) N\left(\tau_{x}(\omega)<\infty ; \omega_{\tau_{x}(\omega)}=x\right) .
$$


(ii) Under $N\left(\cdot \mid \omega_{\bar{g}(\omega)}=x\right)$, the processes $\omega_{\cdot \wedge \bar{g}(\omega)}$ and $\omega \circ \theta_{\bar{g}(\omega)}$ are mutually independent. Furthermore,

- the process $\omega_{\cdot \wedge \bar{g}(\omega)}$ is distributed as $X_{\cdot \wedge \tau_{x}^{\uparrow}}^{\uparrow}$ under $\mathbf{P}\left(\cdot \mid X_{\tau_{x}^{\uparrow}}^{\uparrow}=x\right)$;

- the law of $\omega \circ \theta_{\bar{g}(\omega)}$ is absolutely continuous with respect to the law of $X_{\cdot \wedge \tau_{-x}^{\downarrow}}^{\downarrow}$ and the corresponding density is $\varphi\left(X_{\tau_{-x}^{\downarrow}}^{\downarrow}\right)$, where

$$
\frac{1}{\varphi(y)}=\mathcal{U}^{*}([0,-y)) N^{*}\left(\tau_{-x}(\omega)<\infty\right), \quad y \in(-\infty, 0) .
$$

Remark. - The spectrally positive case is due to Chaumont in [9] or [7].

Proof. - Let $G$ and $D$ be two nonnegative measurable functionals on $\Omega$. From Proposition 4.7(i) and the corresponding dual equality, we get for any $x>0$,

$$
\mathbf{E}\left[G\left(X_{\cdot \wedge \tau_{x}^{\uparrow}}^{\uparrow}\right)\right]=N\left(G\left(\omega_{\cdot \wedge \tau_{x}(\omega)}\right) \mathcal{U}\left(\left(-\omega_{\tau_{x}(\omega)}, 0\right]\right) ; \tau_{x}(\omega)<\infty\right)
$$

and

$$
\mathbf{E}\left[D\left(X_{\cdot \wedge \tau_{-x}^{\downarrow}}^{\downarrow}\right)\right]=N^{*}\left(D\left(\omega_{\cdot \wedge \tau_{-x}(\omega)}\right) \mathcal{U}^{*}\left(\left[0,-\omega_{\tau_{-x}(\omega)}\right)\right) ; \tau_{-x}(\omega)<\infty\right) .
$$

The various assertions of the theorem then follow from the identity

$$
\begin{aligned}
N & (G(\omega \cdot \wedge \bar{g}(\omega) \\
\quad & \left.\frac{1}{d^{*}} \int_{0}^{+\infty} d x N(\omega \circ \theta \bar{g}(\omega))\right)
\end{aligned}
$$

which we now prove.

Let $a$ and $b$ be two positive real numbers. Observe that

$$
\left\{a \leqslant \omega_{\bar{g}(\omega)}<a+b\right\}=\left\{\tau_{a}(\omega)<\infty\right\} \cap\left\{\sup \omega \circ \theta_{\tau_{a}(\omega)}<b+a-\omega_{\tau_{a}(\omega)}\right\} .
$$

On this event, $\omega \circ \theta_{\bar{g}(\omega)}$ is the post-supremum process of $\omega \circ \theta_{\tau_{a}(\omega)}$. Hence, by the Markov property under $N$ at $\tau_{a}(\omega)$, we have

$$
\begin{gathered}
N\left(G\left(\omega_{\cdot \wedge \tau_{a}(\omega)}\right) D\left(\omega \circ \theta_{\bar{g}(\omega)}\right) ; a \leqslant \omega_{\bar{g}(\omega)}<a+b\right) \\
\quad=N\left(G\left(\omega_{\cdot \wedge \tau_{a}(\omega)}\right) d\left(\omega_{\tau_{a}(\omega)}\right) ; \tau_{a}(\omega)<\infty\right),
\end{gathered}
$$

where for any positive number $x$,

$$
d(x)=\mathbf{E}\left[D\left(\vec{X}^{\tau_{-x}}\right) ; S_{\tau_{-x}}<b+a-x\right] .
$$

Let us write $d(x)$ in a more suitable form. First, observe that

$$
d(x)=\mathbf{E}\left[\sum_{j \in \mathcal{I}^{*}} \mathbf{1}_{\left\{X_{g_{j}}<b+a-x ; I_{g_{j}}>-x\right\}} D\left(\omega_{\cdot \wedge \tau_{-x-X_{g_{j}}}^{j}\left(\omega^{j}\right)}\right) \mathbf{1}_{\left\{\tau_{-x-X_{g_{j}}}\left(\omega^{j}\right)<\infty\right\}}\right] .
$$


Then, apply the compensation formula to get

$$
\left.\left.\left.d(x)=\mathbf{E}\left[\int_{0}^{\infty} d u \mathbf{1}_{\left\{X _ { L _ { u } ^ { * - 1 } < b + a - x ; I _ { L _ { u } ^ { * - 1 } } > - x \} } N ^ { * } \left(D \left(\omega_{\cdot \wedge \tau_{-x-X}}{ }_{L_{u}^{*-1}}(\omega)\right.\right.\right.}\right) \mathbf{1}_{\left\{\tau_{-x-X} L_{u}^{*-1}\right.}(\omega)<\infty\right\}\right)\right] .
$$

Set for any real numbers $x$ and $y$,

$$
v(x, y)=\mathbf{1}_{[0,+\infty)}(x) \mathbf{1}_{[0,+\infty)}(y) u^{*}(y) \mathbf{P}\left(I_{\tau_{y}}>-x \mid X_{\tau_{y}}=y\right) .
$$

Then, for any $a \leqslant x<a+b$, Lemma 2.1 implies

$$
\begin{aligned}
d(x) & =\int_{0}^{a+b-x} d y u^{*}(y) \mathbf{E}\left[\mathbf{1}_{\left\{I_{\tau_{y}}>-x\right\}} N^{*}\left(D\left(\omega \cdot \wedge \tau_{-x-y}(\omega)\right) ; \tau_{-x-y}(\omega)<\infty\right) \mid X_{\tau_{y}}=y\right] \\
& =\int_{a}^{a+b} d y v(x, y-x) N^{*}\left(D\left(\omega \cdot \wedge \tau_{-y}(\omega)\right) ; \tau_{-y}(\omega)<\infty\right) .
\end{aligned}
$$

Thus,

$$
\begin{aligned}
& N\left(G\left(\omega \cdot \wedge \tau_{a}(\omega)\right) D(\omega \circ \theta \bar{g}(\omega)) ; a \leqslant \omega_{\bar{g}(\omega)}<a+b\right) \\
& =\int_{a}^{a+b} d y N\left(\mathbf{1}_{\left\{\tau_{a}(\omega)<\infty\right\}} v\left(\omega_{\tau_{a}(\omega)}, y-\omega_{\tau_{a}(\omega)}\right) G\left(\omega_{\left.\cdot \wedge \tau_{a}(\omega)\right)}\right)\right) \\
& \quad \times N^{*}\left(D\left(\omega_{\cdot \wedge \tau_{-y}(\omega)} ; \tau_{-y}(\omega)<\infty\right)\right) .
\end{aligned}
$$

Next, set for any positive integer $n$,

$$
m_{n}=\frac{\left[2^{n} \omega \bar{g}(\omega)\right]}{2^{n}} \quad \text { and } \quad y_{n}=\frac{\left[2^{n} y\right]}{2^{n}}, \quad y \geqslant 0 .
$$

We apply (25) with $a=i 2^{-n}$ and $b=2^{-n}$ for every integer $i \geqslant 0$, and we sum over $i$. It follows that

$$
\begin{aligned}
N( & \left.G\left(\omega \cdot \wedge \tau_{m_{n}}(\omega)\right) D(\omega \circ \theta \bar{g}(\omega))\right) \\
= & \int_{0}^{+\infty} d y N\left(\mathbf{1}_{\left\{\tau_{y_{n}}(\omega)<\infty\right\}} v\left(\omega_{\tau_{y_{n}}(\omega)}, y-\omega_{\tau_{y_{n}}(\omega)}\right) G\left(\omega \cdot \wedge \tau_{y_{n}}(\omega)\right)\right) \\
& \times N^{*}\left(D\left(\omega_{\cdot \wedge \tau_{-y}(\omega)} ; \tau_{-y}(\omega)<\infty\right)\right) .
\end{aligned}
$$

Let $\varepsilon, A>0$. It is sufficient to prove (24) for

$$
G(\omega)=\mathbf{1}_{\{\zeta(\omega)>\varepsilon ; \sup \omega \leqslant A\}} F(\omega) \quad \text { and } \quad D(\omega)=\mathbf{1}_{\{\zeta(\omega)>\varepsilon\}} K(\omega)
$$

where $F(\omega)$ and $K(\omega)$ depend continuously on the values of $\omega$ at some finitely many positive times. Assumption (A) implies that $X$ attains continuously its supremum on any 
finite time interval (see Millar [15]). So does the excursion above the infimum. Thus, $\tau_{m_{n}}(\omega)$ increases to $\bar{g}(\omega)$ when $n$ goes to infinity $N$-almost everywhere. Then,

$$
\lim _{n \rightarrow \infty} \mathbf{1}_{\left\{\tau_{m_{n}}(\omega)>\varepsilon ; m_{n} \leqslant A\right\}}=\mathbf{1}_{\{\bar{g}(\omega)>\varepsilon ; \sup \omega \leqslant A\}}, \quad N \text {-a.e. }
$$

Since $N(\bar{g}(\omega)>\varepsilon ; \sup \omega \leqslant A) \leqslant N(\zeta(\omega)>\varepsilon)<\infty$, dominated convergence applies in the left side of (26) and we get:

$$
\begin{aligned}
& \lim _{n \rightarrow \infty} N\left(G\left(\omega_{\cdot \wedge \tau_{m_{n}}(\omega)}\right) D\left(\omega \circ \theta_{\bar{g}(\omega)}\right)\right) \\
& =N\left(F(\omega \cdot \wedge \bar{g}(\omega)) K\left(\omega \circ \theta_{\bar{g}(\omega)}\right) ; \sup \omega \leqslant A ;(\zeta-\bar{g}(\omega)) \wedge \bar{g}(\omega)>\varepsilon\right) .
\end{aligned}
$$

We now turn to the limit of the right hand side of (26): Recall (2) from Section 2.1

$$
\mathbf{P}\left(\exists t \in(0,+\infty): S_{t-}=X_{t-}<X_{t}\right)=0 .
$$

It implies that for any positive number $y, N\left(\omega_{\tau_{y}(\omega)-}=y<\omega_{\tau_{y}(\omega)}\right)=0$. We also recall that $\omega(0)=0, N$-a.e. Thus,

$$
N \text {-a.e. } \quad \lim _{n \rightarrow \infty} \mathbf{1}_{\left\{\omega_{\tau_{y_{n}}(\omega)} \leqslant y\right\}}=\mathbf{1}_{\left\{\omega_{\tau y}(\omega)=y\right\}} .
$$

Next, for any $x>0, \lim _{\varepsilon \rightarrow 0} v(x, \varepsilon)=u^{*}(0+)=\frac{1}{d^{*}}$ because

$$
0 \leqslant d^{*} u^{*}(\varepsilon)-d^{*} v(x, \varepsilon)=d^{*} u^{*}(\varepsilon) \mathbf{P}\left(I_{\tau_{\varepsilon}} \leqslant-x \mid X_{\tau_{\varepsilon}}=\varepsilon\right) \leqslant \mathbf{P}\left(I_{\tau_{\varepsilon}} \leqslant-x\right) \underset{\varepsilon \rightarrow 0}{\longrightarrow} 0 .
$$

Thanks to (28) we get $N$-a.e.

$$
\lim _{n \rightarrow \infty} \mathbf{1}_{\left\{\tau_{y_{n}}(\omega)<\infty\right\}} v\left(\omega_{\tau_{y_{n}}(\omega)}, y-\omega_{\tau_{y_{n}}(\omega)}\right)=u^{*}(0+) \mathbf{1}_{\left\{\omega_{\tau_{y}(\omega)}=y\right\}}=\frac{1}{d^{*}} \mathbf{1}_{\left\{\omega_{\tau_{y}(\omega)}=y\right\}} .
$$

Observe that $N$-a.e. on $\left\{\omega_{\tau_{y}(\omega)}=y\right\}, \tau_{y_{n}}(\omega)$ increases towards $\tau_{y}(\omega)$. Therefore, by dominated convergence

$$
\begin{aligned}
& \lim _{n \rightarrow \infty} N\left(\mathbf{1}_{\left\{\tau_{y_{n}}(\omega)<\infty\right\}} v\left(\omega_{\tau_{y_{n}}(\omega)}, y-\omega_{\tau_{y_{n}}(\omega)}\right) G\left(\omega_{\cdot \wedge \tau_{y_{n}}(\omega)}\right)\right) \\
& \quad=\frac{1}{d^{*}} \mathbf{1}_{(0, A]}(y) N\left(F\left(\omega_{\cdot \wedge \tau_{y}(\omega)}\right) ; \varepsilon<\tau_{y}(\omega)<\infty ; \omega_{\tau_{y}(\omega)}=y\right) .
\end{aligned}
$$

However, for any $y>0$, we have

$$
\begin{aligned}
& N\left(\mathbf{1}_{\left\{\tau_{y_{n}}(\omega)<\infty\right\}} v\left(\omega_{\tau_{y_{n}}(\omega)}, y-\omega_{\tau_{y_{n}}(\omega)}\right) G\left(\omega \cdot \wedge \tau_{y^{*}}(\omega)\right)\right) N^{*}\left(D\left(\omega_{\cdot \wedge \tau_{-y}(\omega)} ; \tau_{-y}(\omega)<\infty\right)\right) \\
& \quad \leqslant M^{2} \mathbf{1}_{(0, A]}(y) \sup _{x \in\left(0,2^{-n}\right]} u^{*}(x) N(\zeta>\varepsilon) N^{*}(\zeta>\varepsilon),
\end{aligned}
$$

where $M$ is a bounding constant of $F$ and $K$. Then, dominated convergence applies in the right side of (26), which yields the desired identity (24) thanks to (25).

\section{REFERENCES}

[1] J. Bertoin, Sur la décomposition de la trajectoire d'un processus de Lévy spectralement positif en son minimum, Ann. Inst. H. Poincaré. 27 (4) (1991) 537-547. 
[2] J. Bertoin, An extension of Pitman's theorem for spectrally negative Lévy processes, Ann. Probab. 20 (1992) 1464-1483.

[3] J. Bertoin, Splitting at the infimum and excursions in half-lines for random walks and Lévy processes, Stoch. Process. Appl. 47 (1993) 17-45.

[4] J. Bertoin, Lévy Processes, Cambridge Univ. Press, 1996.

[5] N.H. Bingham, Fluctuation theory in continuous time, Adv. Appl. Probab. 7 (1975) 705766.

[6] J.M. Bismut, Last exit decomposition and regularity at the boundary of transition probabilities, Zeitschrift Wahr. 69 (1985) 65-98.

[7] L. Chaumont, Processus de Lévy et conditionnement, Thèse de doctorat, Laboratoire de Probabilités de Paris 6, 1994.

[8] L. Chaumont, Sur certains processus de Lévy conditionnés à rester positifs, Stochastics and Stochastics Reports 47 (1994) 1-20.

[9] L. Chaumont, Conditionings and paths decompositions for Lévy processes, Stochastic Process. Appl. 64-1 (1996) 39-54.

[10] L. Chaumont, Excursion normalisée, méandre et pont pour les processus de Lévy stables, Bull. Sci. Math. 121-5 (1997) 377-403.

[11] H. Kesten, Hitting probabilities of a single point for processes with stationary independent increments, Mem. Amer. Math. Soc. 93 (1969).

[12] J.-F. Le Gall, Y. Le Jan, Branching processes in Lévy processes: the exploration process, Ann. Probab. 26-1 (1998) 213-252.

[13] Y. Le Jan, Dual Markovian Semigroups and Processes, in: Functional Analysis in Markov Processes, in: Lect. Notes Math., Vol. 923, Springer-Verlag, 1981.

[14] P.W. Millar, Exit properties of stochastic processes with independent increments, Trans. Amer. Math. Soc. 178 (1973) 459-479.

[15] P.W. Millar, Exit zero-one laws and the minimum of a Markov process, Trans. Amer. Math. Soc. 226 (1977) 365-391.

[16] J. Pitman, One-dimensional Brownian motion and the three-dimensional Bessel process, Adv. Appl. Probab. 7 (1975) 511-526.

[17] L.C.G. Rogers, A new identity for real Lévy processes, Ann. Inst. H. Poincaré 20 (1984) 21-34.

[18] M.L. Silverstein, Classification of coharmonic and coinvariant functions for a Lévy process, Ann. Probab. 8 (1980) 539-575.

[19] D. Williams, Path decomposition and continuity of local time for one-dimensional diffusion, Proc. London Math. Soc. 28 (1974) 738-768. 